\title{
Avoiding unseen obstacles: \\ Subcortical vision is not sufficient to maintain normal obstacle avoidance behaviour during reaching
}

Alasdair I. Ross ${ }^{1}$, Thomas Schenk ${ }^{2}$, Jutta Billino ${ }^{3}$, Mary Joan Macleod ${ }^{4} \&$ Constanze Hesse $^{1 *}$

$14 / 09 / 2016$

Running head: Obstacle avoidance in hemianopia

*Correspondence should be addressed to:

Dr Constanze Hesse

School of Psychology

University of Aberdeen

William Guild Building (Room T11)

Aberdeen AB24 3FX

United Kingdom

Phone: +44 (0)1224 273215

E-mail: c.hesse@abdn.ac.uk

${ }^{1}$ School of Psychology, University of Aberdeen, Aberdeen, United Kingdom

${ }^{2}$ Clinical Neuropsychology, Ludwig-Maximilians-Universität München, Germany

${ }^{3}$ Experimental Psychology, Justus-Liebig-Universität, Giessen, Germany

${ }^{4}$ School of Medicine \& Dentistry, University of Aberdeen, Aberdeen, United Kingdom 


\section{Abstract}

Previous research found that a patient with cortical blindness (homonymous hemianopia) was able to successfully avoid an obstacle placed in his blind field, despite reporting no conscious awareness of it (Striemer, C. L., Chapman, C. S., \& Goodale, M. A., 2009, PNAS, 106(37), 15996-16001). This finding led to the suggestion that dorsal stream areas, that are assumed to mediate obstacle avoidance behaviour, may obtain their visual input primarily from subcortical pathways. Hence, it was suggested that normal obstacle avoidance behaviour can proceed without input from the primary visual cortex. Here we tried to replicate this finding in a group of patients $(\mathrm{N}=6)$ that suffered from highly circumscribed lesions in the occipital lobe (including V1) that spared the subcortical structures that have been associated with action-blindsight. We also tested if obstacle avoidance behaviour differs depending on whether obstacles are placed only in the blind field or in both the blind and intact visual field of the patients simultaneously. As expected, all patients successfully avoided obstacles placed in their intact visual field. However, none of them showed reliable avoidance behaviour - as indicated by adjustments in the hand trajectory in response to obstacle position - for obstacles placed in their blind visual field. The effects were not dependent on whether one or two obstacles were present. These findings suggest that behaviour in complex visuomotor tasks relies on visual input from occipital areas.

Keywords: hemianopia, action-blindsight, dorsal stream, V1, visual perception 


\section{Introduction}

In our day-to-day lives we frequently have to avoid obstacles in our surroundings, such as reaching out for a cup of coffee on a cluttered desk or avoiding other pedestrians while walking along a busy street. Even though successful obstacle avoidance relies on a complex interplay between the visual and the visuomotor systems, it appears to be an automatic process that does not require much conscious consideration (Chapman \& Goodale, 2008). From a neuropsychological perspective, it has been suggested that obstacle avoidance is a prime example of a pure dorsal stream function (McIntosh, McClements, Dijkerman, Birchall, \& Milner, 2004; McIntosh, McClements, Schindler, et al., 2004; Rice et al., 2008; Rice et al., 2006; Schindler et al., 2004). This suggestion relates back to the predictions of the perception-action model (Milner \& Goodale, 1995, 2006, 2008) according to which visual information is processed in anatomically and functionally distinct pathways depending on the purpose it is used for. Specifically, the model suggests that visual information used for object identification and recognition is processed in the ventral cortical pathway (vision for perception) while visual information used for action control such as reaching, grasping and obstacle avoidance are processed in the dorsal cortical pathway (vision for action). Furthermore, the model suggests that the visual information processed within both pathways shows different properties. The visual information processed in the ventral stream is assumed to be consciously available, stored over long time periods and represented within an allocentric frame of reference. In contrast, the visual information within the dorsal stream is processed unconsciously, in real-time, and is represented within an egocentric frame of reference (Milner \& Goodale, 1995, 2006, 2008).

Evidence for both claims, i.e. that a) obstacle avoidance is a dorsal stream function that can b) occur without conscious awareness comes primarily from neuropsychological studies. In a series of experiments it was shown that patients with damage to the ventral cortical pathways show normal obstacle avoidance behaviour and adjust their movement paths in response to the positions of obstacles in the workspace (McIntosh, McClements, Dijkerman, et al., 2004; Rice et al., 2006). In contrast, patients with damage to dorsal stream areas (suffering from optic ataxia) show impaired obstacle avoidance behaviour, being unable to adjust their hand movements in response to obstacle position (Rice et al., 2008; Schindler et al., 2004). These findings support the idea that obstacle avoidance behaviour relies on visual guidance provided by the dorsal stream. Moreover, 
in accordance with the notion that conscious awareness is not necessary to show normal obstacle avoidance performance, McIntosh, McClements, Schindler, et al. (2004) showed that a patient suffering from visual extinction after lesion to his ventral stream adjusted his hand movements in response to obstacle position even when he was unable to report whether obstacles were present or not.

As this research indicates that obstacle avoidance behaviour can be unaffected by deficits in conscious vision, the question arises of where the visual inputs into the dorsal system originate from. According to the perception-action model (Milner \& Goodale, 1995, $2006,2008)$ it is assumed that both the dorsal and ventral stream obtain their visual input primarily from area V1. However, more recently, it has been argued that obstacle avoidance behaviour can actually proceed normally without input from V1 (Striemer, Chapman, \& Goodale, 2009) implying that some functions in the dorsal stream primarily rely on visual information mediated via subcortical pathways. The basis for this suggestion was the observation that a patient suffering from hemianopia, due to damage in the primary visual cortex, was able to successfully avoid obstacles placed in his blind visual field despite being completely unaware of their presence (Striemer et al., 2009). This finding indicates that obstacle-avoidance behaviour can possibly be guided by subcortical vision alone, meaning that there must exist a direct processing route to the dorsal parietal cortex bypassing the primary visual pathway from LGN to V1. In fact, human and primate lesion studies have provided evidence that the dorsal pathways obtain (functionally relevant) input from several subcortical structures via the retinotectal-pulvinar (RTP) pathway (Bridge, Leopold, \& Bourne, 2016; Danckert \& Rossetti, 2005; Kaas \& Lyon, 2007; Lyon, Nassi, \& Callaway, 2010; Rodman, Gross, \& Albrigh, 1990; Stoerig \& Cowey, 2007; Tamietto et al., 2009; Zihl, 1980). The RTP-pathway passes through the pulvinar and projects to extrastiate areas. RTP projections are supposed to reach dorso-parietal areas that are known to mediate visuomotor behaviour via area V5/MT. This pathway has frequently been associated with the action-blindsight phenomenon (Danckert \& Rossetti, 2005). The term blindsight refers to the observation that some patients with lesions to V1 can produce above chance performance in their blind visual field when performing visual tasks such as colour or orientation discrimination (Stoerig, 1993; Stoerig \& Cowey, 1997) as well as visuomotor tasks such as reaching or grasping (Danckert et al., 2003; Danckert \& Rossetti, 2005; Jackson, 1999; 
Perenin \& Jeannerod, 1975; Perenin \& Rossetti, 1996; Weiskrantz, Warrington, Sanders, \& Marshall, 1974; Whitwell, Striemer, Nicolle, \& Goodale, 2011).

Based on the finding that their patient showed an action-blindsight phenomenon, in an obstacle avoidance task, Striemer and colleagues hypothesised that the primitive subcortical pathways may, in general, play a more crucial role in controlling complex visuomotor behaviour than originally thought (Striemer et al., 2009, p. 5). Importantly, however, their patient (CB) only showed normal visuomotor performance in an obstacle avoidance task but was unable to point to targets presented in his blind field (localisation task). The finding that the patient was able to act normally in some, but not all manual action-tasks suggests that the subcortical route is restricted to very specific types of visuomotor behaviour. As Striemer et al.'s hypothesis is based on a single-case study, both aspects of their proposal, i.e., the anatomical assertion that the origin of the visual information used for obstacle-avoidance is subcortical; and the functional assertion that obstacle-avoidance but not pointing behaviour is served by this pathway, are open for debate. The issue related to the anatomical aspect of this hypothesis is that in addition to the subcortical visual structures also large portions of the early visual cortex were undamaged in patient CB. Consequently, it is possible that CB's blindsight performance was mediated by visual information originating from extrastriate visual cortex areas (such as V2 and V3). If this was the case, we would expect that other patients with more extensive damage to the extrastriate occipital cortex would fail to show intact obstacleavoidance behaviour. If, however, intact obstacle behaviour merely relies on subcortical input, the extent to which the occipital cortex is damaged should have no relevance. A similar uncertainty holds for the functional aspect of Striemer et al.'s hypothesis. Again, because of the single-case nature of the observation, it is not clear whether the dissociation between pointing performance and obstacle-avoidance performance is specific to their patient CB or whether it is indeed a pattern that occurs reliably whenever $\mathrm{V} 1$ is impaired but subcortical areas of the visual systems are intact. Only in the latter case, we can conclude that distinct visual pathways serve obstacle avoidance and pointing.

Additionally, it is important to point out that, if as suggested, obstacle avoidance behaviour is primarily mediated by subcortical visual structures also in the intact brain, then patients' performance in their blind field should be indistinguishable from their performance shown in the intact visual field, and also similar to that of neurologically 
healthy participants. For perceptual blindsight it has been shown that even though patients show performance levels that are above chance they are still performing considerably worse than in their intact visual field and also much worse than neurologically healthy participants (e.g., Azzopardi \& Cowey, 1997; Morland et al., 1999; Ptito, Lassonde, Leporé, \& Ptito, 1987; Ptito, Lepore, Ptito, \& Lassonde, 1991). For action-blindsight this is less clear as performance cannot as easily be classified as being "correct" or "incorrect". Notably, while Striemer and colleagues (2009) provided evidence that their patient could avoid a single object placed in his blind visual field, they did not directly test whether the patient's ability was in fact normal (i.e. indistinguishable from that of healthy participants).

To address these open questions, we examined a group of highly functional patients with selective lesions to the occipital cortex (see Figure 1 and Table 1). We measured their pointing and obstacle avoidance performance in both their blind and their seeing visual hemifields and compared their performance to a matched group of neurologically intact control participants. Moreover, we aimed to address another issue related to the study conducted by Striemer and colleagues (2009). In their study, both a single-obstacle avoidance paradigm (placing only one obstacle in one of the visual fields) as well as a two-obstacle avoidance paradigm (placing obstacles simultaneously in both visual fields) were used, but only the findings from the single-obstacle paradigm were presented in detail. The reason for this is that in their two-obstacle condition the positions of both obstacles were changed simultaneously in the blind and sighted visual fields of the patient making it impossible to tell whether the effects were a) different from those observed for the one-obstacle task and b) similar for obstacle shifts occurring in the blind and sighted visual field in this condition. This confound can, however, easily be avoided. Furthermore, we think that the two-obstacle paradigm is the more appropriate task as it may provide a more sensitive indicator of a patient's ability to use visual information from their blind field for movement planning and control than the singleobstacle task. In the single obstacle version the entire space opposite the obstacle is free of obstacles and therefore there is no reason for why participants should not simply direct their hand to this empty space thereby keeping a large distance from any obstacle placed in one half of the workspace. However, the further the hand is moved away from an obstacle the less likely it becomes that obstacle position will still affect movement path selection. In contrast, when two obstacles are placed into the two halves of the workspace the optimal strategy would be to find a movement path that passes midway 
between the two obstacles, thereby minimising the risk of collision with any of them. Importantly, this strategy requires participants to take the position of both obstacles into account. Hence, we think that a two-obstacle paradigm may provide a more sensitive task to detect a patient's ability to use visual information from their blind field for obstacle avoidance.

For that reason, we examined our patients in both a single-obstacle and a two-obstacle avoidance paradigm. Incidentally this also afforded us to test if avoidance performance varies depending on whether or not visual information is also available in the intact visual field. This is interesting as previous studies suggest that available conscious vision can facilitate blindsight performance (Jackson, 1999; Ward \& Jackson, 2002) with increased detection rates for stimuli in the blind field when concurrent stimuli are presented in the intact hemifield. Finally, as patient CB had shown a redundant target effect (RTE) as indicated by faster reaction times for stimuli simultaneously presented in the blind and intact visual field than for stimuli presented in the intact field alone, we also tested each patient with the redundant target paradigm (Marzi, Tassinari, Aglioti, \& Lutzemberger, 1986) to investigate whether this indicator of perceptual blindsight can predict the occurrence of action-blindsight. As Striemer and colleagues suggested that obstacle-avoidance may provide a more sensitive and robust measure to assess residual visual processing in patients with damage to the primary visual pathways, patients that show a RTE should be likely to also exhibit preserved obstacle-avoidance behaviour.

\section{Methods}

\subsection{Participants}

During the course of the study (2012-2015) we contacted 42 stroke patients who were diagnosed with hemianopia due to lesions in the occipital cortex and who showed no signs of visual neglect (initial contact was made via M.J. Macleod, a senior stroke consultant at the NHS Grampian, Aberdeen). Out of the 42 patients, 30 got in contact with us to obtain further information on the study. Eighteen of those patients then expressed an interest to take part (12 patients decided against participation due to ongoing health issues associated with their stroke). Out of these 18 patients, $10 \mathrm{did}$ not fulfil our criteria as our perimetry revealed a too large area of visual sparing and/or the heminopia had partly resolved since the initial diagnosis. Furthermore, one patient sadly died due to another stroke after contact and one patient withdrew after consent. This left us with a final sample size of six patients ( 2 female, 4 male, age range $=45-72$ years) with homonymous hemianopia or quadranopia who participated in the experiment. All 
patients were right-handed by self-report and had normal or corrected-to-normal visual acuity. None of the patients suffered from any motor difficulties such as hemiplegia or hemiparesis or showed signs of comorbid visuo-spatial neglect as confirmed by their medical reports and the star cancellation task (Halligan, Cockburn, \& Wilson, 1991). All patients were highly functional and had uncompromised language and cognitive abilities to understand and follow the task instructions. For all patients, the visual field deficits were chronic and the consequence of an ischaemic stroke that they had suffered at least 6 months prior to testing (range of lesion onset: 6-14 months, see Table 1). Patient 1 reported some spared sensitivity to motion in her blind visual field (i.e. Riddoch phenomenon) which we confirmed in the lab.

Six neurologically healthy, right-handed, and age ( \pm 5 years) and gender matched adults served as a control group. Once a control participant was identified, their left and right visual fields were matched to those of the corresponding patient. That is, if a patient suffered from left hemianopia, the performance of the matched control in the left field was compared to the patient's performance in their blind field and the performance of the control participant in the right field was compared to the patient's performance in their intact visual field, and vice versa.

All patients performed all tests (perimetry, perceptual tasks, and obstacle avoidance tasks) in at least two different sessions on two different days (within 2 weeks). Two of the patients were tested in three sessions. Each session took about 2-3 hours depending on the number and duration of breaks. The experiment was undertaken with the understanding and written consent of each participant and approved by the NHS Grampian Research Ethics Committee as well as the local ethics committee of the School of Psychology at the University of Aberdeen.

\subsection{Lesion mapping}

The lesion location for each patient was determined based on clinical imaging as available. We had access either to CT scans (3 patients) or to MRT scans (3 patients). The MRIcro software (Rorden \& Brett, 2000) was used for lesion mapping. Patients' lesions were drawn manually onto transversal slices of the publicly available Montreal Neurological Institute (MNI) brain, a T1-weighted template MRT scan, which is oriented to match the Talairach space (Talairach \& Tournoux, 1988). Slices chosen for mapping corresponded to Talairach z-coordinates $60,50,40,32,24,16,8,0,-8,-16$, and $-24 \mathrm{~mm}$ (see Figure 1). Lesion descriptions are provided in Table 1. 
Insert Figure 1 about bere

\subsection{Visual field assessment}

Visual fields were assessed monocularly with a static automated threshold perimetry using a Humphrey Field Analyser (HFA; Carl Zeiss Meditec AG, Oberkocken, Germany). We used two pre-set programmes (for more details see also, Walsh, 2011): The 30-2 SITA-algorithm was used for general screening, and tests each eye separately within $30^{\circ}$ of fixation vertically and horizontally with a total of 76 points (using $6^{\circ}$ spacing with a $3^{\circ}$ offset from the central meridians). To asses macular sparing we tested each eye using the 10-2 SITA-algorithm which tests vision within $10^{\circ}$ of fixation using 68 points ( $2^{\circ}$ grid spacing with an $1^{\circ}$ offset from the central meridians). The central target (yellow light) was used as fixation point and the target stimulus was a supra-threshold white light with a diameter of $0.43^{\circ}$ (Goldmann size III). Furthermore, we performed a binocular Esterman test (Esterman, 1982) to assess patients' full visual field (120 points at a light intensity of $10 \mathrm{~dB}$ testing $75^{\circ}$ nasally, $75^{\circ}$ degrees temporally, $40^{\circ}$ superiorly and $60^{\circ}$ inferiorly). Patients responded to the detection of a target light with a button press. Visual field sparing was defined as the distance between fixation and the border between the detected and undetected stimuli points along the left or right horizontal axes as well as the vertical axes (see Table 1).

\subsection{Tests of perceptual abilities}

\subsubsection{Apparatus and Materials}

All patients were assessed for their ability to process visual information in their blind field. To do so, we used the same two tasks that were previously employed by Striemer et al. (2009): the redundant target effect (Marzi et al., 1986) and a localisation task (Corbetta, Marzi, Tassinari, \& Aglioti, 1990). For both tasks, patients were seated on a height-adjustable chair in front of a 19-inch Iiyama-Prolite touch screen monitor (T1931SR-B1, $1280 \times 1024$ pixel, $60 \mathrm{~Hz}$ ) at a viewing distance of $500 \mathrm{~mm}$. To maintain a constant viewing distance between trials, patients were asked to place their head on a chinrest. Fixation was controlled with an Eyelink II system (SR Research, Ontario, Canada). Pupil location was sampled at a rate of $250 \mathrm{~Hz}$. Fixation performance was monitored online by the experimenter and whenever an eye-movement was observed during a trial, the trial was discarded and repeated later at a random position within the 
experiment. Both experiments were programmed in MATLAB using the Psychophysics Toolbox (Brainard, 1997) and the Eyelink toolbox (Cornelissen, Peters, \& Palmer, 2002). A button box placed on the table was used to record the responses in the redundant target effect task and served as the start position of the hand in the localisation task.

\subsubsection{Procedure}

Task A: Redundant target effect. The task of the patients was to quickly respond with a button press whenever they perceived a target on the screen. Targets were black dots with a diameter of $15 \mathrm{~mm}$ presented for $150 \mathrm{~ms}$ on a uniform grey background. For all patients the targets were presented to the left and/or right of the fixation cross at an eccentricity of $12^{\circ}$ of visual angle. The fixation cross was presented in black $(10 \mathrm{~mm}$ x 10 $\mathrm{mm}$, line width $1 \mathrm{~mm}$ ) centrally on the screen. For patients with quadranopia or macular sparing of $10^{\circ}$ in the superior or inferior blind field the position of the targets relative to the fixation cross was adjusted (i.e. moved upward or downward) such that all target presented in the contralesional field fell within the blind area as determined by the perimetry. Targets could occur in the sighted field only, in the blind field only, in both fields simultaneously, or not be present at all (catch trials). There were 20 trials for each condition which occurred in random order resulting in a total of 80 trials. If patients did not detect a target, and thus did not press the button an audible beep (500 Hz, $100 \mathrm{~ms}$ ) signalled the end of the trial after $1500 \mathrm{~ms}$.

Task B: Localisation Task. This task is a classic visuomotor task that has previously been applied to test for the existence of action-blindsight in patients with hemianopia (e.g., Corbetta et al., 1990; Striemer et al., 2009; Weiskrantz et al., 1974; Whitwell et al., 2011). In this task, patients are asked to point to stimuli presented at different visual eccentricities in their blind and intact visual fields. Again targets were presented as black dots (diameter $15 \mathrm{~mm}$ ) on a uniform grey background at eccentricities of $15^{\circ}, 25^{\circ}$ and $35^{\circ}$ of visual angle. Depending on condition (intact vs. blind field), the fixation cross was presented centrally (in vertical dimension) and $10 \mathrm{~mm}$ from the left or right edge of the screen (in horizontal dimension). All patients were first tested in their intact visual field (so they understood the task) and subsequently in their blind visual field. At the start of the trial, the fixation cross was visible for $1000 \mathrm{~ms}$, then the target appeared for $1000 \mathrm{~ms}$ after which a beep $(1000 \mathrm{~Hz}, 100 \mathrm{~ms})$ signalled patients to point to the position of the target on the touch screen monitor. Pointing movements were performed open-loop 
meaning that the target was removed from the screen when participants started their movement. The distance between start button and the centre of the screen was approximately $400 \mathrm{~mm}$. Each target position was presented 10 times in random order resulting in a total of 30 trials per visual hemifield. When tested in their blind field, patients were asked to make their best guess of where stimuli were presented when pointing to the screen. The targets were presented to the left or right of the fixation cross. For patients with quadranopia the position of the targets relative to the fixation cross was adjusted such that all targets fell within the blind field as determined by the perimetry (targets in the intact field were presented at the mirror symmetric position).

Note that only patients were tested in the RTE-task and the localisation task but not the control participants. The main reason for using these tasks was to determine if patients who show some kind of residual visual processing will also show preserved obstacleavoidance behaviour. We used both a classic visuomotor blindsight task (i.e. localisation) and a task that has been considered to provide a quite conservative measure for the existence of perceptual blindsight (i.e. the RTE-task). According to previous studies spatial interfield summation of visual stimuli, as indicated by the RTE, is relatively rare in hemianopic patients (Marzi et al., 1986) and harder to find than residual spatial localisation abilities (Corbetta et al., 1990). Testing our patients in these conventional blindsight tasks is critical as it was suggested that obstacle-avoidance may constitute a more robust and sensitive measure for the existence of residual-visual processing than any other blindsight phenomenon (Striemer et al., 2009).

\subsection{Obstacle avoidance tasks}

All patients and control participants took part in two obstacle avoidance tasks: a singleobstacle task and a two-obstacle task (see Figure 2).

\subsubsection{Apparatus and Materials}

Participants sat on a height-adjustable chair within a lit room. A grey LEGO Baseplate (600 mm x $600 \mathrm{~mm}$ ) was secured on top of a table and used to present the stimuli. The start position consisted of a single black LEGO block $(10 \mathrm{~mm}$ x $10 \mathrm{~mm}$ x $10 \mathrm{~mm})$ that was aligned vertically to the midline of the participant's body and horizontally with the middle of the board. Participants were asked to keep their hand upright with the tip of the fourth finger placed on top of the start position. A chinrest was used to maintain a constant head position throughout the experiment. 
A strip of yellow card $(495 \mathrm{~mm} \times 110 \mathrm{~mm})$ marked the target zone that was located at a straight-line distance of $360 \mathrm{~mm}$ from the hand's starting position. The obstacles were placed along a virtual horizontal line $200 \mathrm{~mm}$ in front of the start position. The obstacles were made of 15 black LEGO blocks (10 mm x $10 \mathrm{~mm}$ x $10 \mathrm{~mm}$ ). They could be placed at two positions on the board either $100 \mathrm{~mm}$ to the right or left from the midline or 60 $\mathrm{mm}$ to the left or right of the midline. Central fixation points were centrally aligned to the start position and marked out on the setup using coloured circular stickers (diameter: $10 \mathrm{~mm}$ ). The position of fixation points was dependent on a patient's area of blindness (as determined by perimetry). Four of the patients fixated at a position $130 \mathrm{~mm}$ (P3, P4 and P6) or $240 \mathrm{~mm}$ (P5) above the target zone that was marked on a wooden board (495 $\mathrm{mm}$ x $285 \mathrm{~mm}$ ) located directly behind the target zone (see Figure 2). Two patients (P1 and P2) were asked to fixate on the board at a position located $45 \mathrm{~mm}$ from the start position (placing the obstacles in their upper blind field). The control participants used the same fixation spots as used by the matching patient.

\section{Insert Figure 2 about here}

Hand position was recorded using an infrared-based Optotrak 3020 system (Northern Digital Incorporation, Waterloo, Ontario, Canada) at a sampling frequency of $200 \mathrm{~Hz}$. Three infrared light emitting diodes (IREDs) were attached to the right hand: at the nail of the thumb, the tip of the index finger and the wrist (for illustration see Fig. 1b in Ross, Schenk, \& Hesse, 2014). Prior to running the experiment, the plane of the obstacle board was calibrated to the Cartesian $(\mathrm{x}, \mathrm{y}, \mathrm{z})$ coordinate system with the start position being set to the origin of the coordinate system. The experiment was programmed using MATLAB and the custom-built Optotrak Toolbox (Franz, 2004).

We monitored fixation with a BlueGain Electro-oculogram (EOG) amplifier (Cambridge Research Systems. Kent, England). Two electrodes were placed around the left eye with one being attached above right edge of the left eyebrow (top right of the superior orbital margin) and the other one below the eye toward the left temple (near the outer canthus). An additional earth electrode was attached to the left earlobe. Pilot-tests had indicated that with this setup we could reliably detect vertical and horizontal eye-movements if they exceeded about 2 degrees of visual angle resulting in voltage changes between 10-20 microVolts. EOG and Optotrak were synchronised using an infrared signal transmitted 
to the EOG at the beginning and end of each trial. The EOG data was monitored in real-time by the experimenter. If a participant failed to maintain fixation the trial was discarded and repeated at a random position within the experiment. On average participants lost fixation in about $6 \%$ of all trials.

\subsubsection{Procedure}

All patients and control participants performed two obstacle avoidance tasks in which either one obstacle was placed at the left or right of the movement path (single obstacle task) or two obstacles were placed simultaneously at either side of the midline (two obstacle task).

Single Obstacle Task: In this task the obstacle could be placed at four different positions: 60 or $100 \mathrm{~mm}$ to the left or right of the midline. Furthermore, to prevent patients from assuming that there would always be an obstacle in their blind field if they did not perceive one, we also included trials in which we placed no obstacle on the board. Each condition was tested 12 times occurring in random order (60 trials in total).

Two Obstacle Task: In this task, two obstacles could be placed in three different configurations on the board (left obstacle in $(60 \mathrm{~mm})$ and right obstacle out $(100 \mathrm{~mm})$; right obstacle in and left obstacle out, and both obstacles out). Thus, gap sizes were either $200 \mathrm{~mm}$ when both obstacles were placed at the out position or $160 \mathrm{~mm}$ when one of the obstacles was at the inner position. Each condition was tested 12 times and presented in random order (36 trials in total).

At the start of each trial participants positioned their right hand at the start position and were asked to close their eyes. The experimenter then arranged the obstacles and manually started the trial with a key press. Subsequently, an auditory signal $(500 \mathrm{~Hz}, 100$ ms) indicated for participants to open their eyes and to fixate at the fixation position. Following a $1500 \mathrm{~ms}$ preview period another beep (1000 Hz, $100 \mathrm{~ms}$ ) signalled participants' to start their reaching movement and to quickly move their hand between the obstacles into the target zone, touch the yellow cardboard, and move back to the start position. Participants were instructed to maintain fixation at the fixation position throughout their movement and to keep looking at the direction of the fixation point when shutter glasses were closed between trials. After $3000 \mathrm{~ms}$ the Optotrak stopped 
sampling and participants were instructed to close their eyes again so that the experimenter could prepare the next trial. At the end of each trial, we prompted patients to report if they had any awareness of an obstacle present in their blind field.

\subsection{Data Analysis}

The 3D position data of all markers was used to compute the resultant movement velocity. Movement onset was defined at the moment one of the markers exceeded a velocity threshold of $0.05 \mathrm{~m} / \mathrm{s}$. The end of movement was defined at the point at which the velocity of one of the markers dropped below a threshold of $0.1 \mathrm{~m} / \mathrm{s}$. Movement times (MTs) were calculated as the time between movement onset and end of the movement. To determine the hand position throughout the obstacle avoidance task we calculated the virtual midpoint (VM) between the three infrared markers in 3D (for a similar procedure see Hesse, Lane, Aimola, \& Schenk, 2012).

Trajectories of the VM were normalised by dividing the data into 100 equal time intervals (using linear interpolation) between movement onset and end of movement. Additionally, we determined the lateral position (x-direction) of the VM at the moment the VM passed between the obstacles in y-direction (Ross et al., 2014). In accordance with previous studies, we computed the sensitivity to obstacle shifts occurring in the left and right visual field respectively (McIntosh, McClements, Dijkerman, et al., 2004; McIntosh, McClements, Schindler, et al., 2004; Rice et al., 2008; Rice et al., 2006; Schindler et al., 2004). These values measure the average lateral shift in hand position in response to a change of obstacle position from $100 \mathrm{~mm}$ to $60 \mathrm{~mm}$ from the midline at either side. That is, sensitivity to obstacle shifts was calculated separately for each side (left and right) by subtracting the lateral hand position (at the moment the obstacles were passed) when the obstacle was placed at an outer position from the hand position measured when the obstacle was placed at an inner position. Note, that in line with previous studies (McIntosh, McClements, Dijkerman, et al., 2004; McIntosh, McClements, Schindler, et al., 2004; Rice et al., 2008; Rice et al., 2006; Schindler et al., 2004; Striemer et al., 2009) we did not analyse the no-obstacle condition in the singleobstacle task. This condition was primarily introduced to prevent the patients from adopting any kind of strategies, as otherwise they would know that if they saw no obstacle it would be located in their blind field. All participants were informed that there were trials in which no obstacle was present. 
Sensitivity to obstacle shifts and MTs were analysed on a group level using an ANOVA with visual field (blind, sighted) as within-subjects factor and group (patient, control) as a between subjects factor. Independent samples t-tests were applied as post-hoc comparisons to compare group differences. Furthermore, to determine if any individual patient showed some positive evidence for preserved obstacle-avoidance, we also determined for each patient separately if obstacle position (in vs. out) affected the lateral hand position across trials in the blind and in the intact visual field using independent samples t-tests across all trials in both obstacle conditions. A significance level of $\alpha=$ 0.05 was used for all analyses and two-tailed p-values are reported if not stated otherwise.

\section{Results}

\subsection{Perceptual tests}

Redundant target effect (RTE): We tested for the occurrence of a RTE on both a group level as well as for every individual patient. The aim of the latter analysis was to determine whether or not the presence of a RTE in a patient predicts the occurrence of other blindsight phenomena. Average reaction times (RTs) for each patient to stimuli presented only in the sighted field or in both the blind and sighted field simultaneously can be seen in Fig. 3a. A paired-samples t-test on the group level revealed no significant difference between RTs for the presentation of one and two targets respectively, $t(5)=1.01, p=.36$. On average patients were about $12.4 \mathrm{~ms} \pm 12.2 \mathrm{~ms}$ faster when targets were presented in both hemifields simultaneously as compared to trials in which the target was only visible in their sighted field. On an individual level, paired samples t-tests further confirmed that for five of the patients there was no significant difference between RTs in both conditions (all $\mathrm{p}>$.52). However, for P4 there was evidence for a redundant target effect with faster RTs when stimuli were present in the sighted and blind field simultaneously (339 ms $\pm 15 \mathrm{~ms}$ ) as compared to the sighted field only (397 $\mathrm{ms} \pm 23 \mathrm{~ms}), \mathrm{t}(19)=2.36, \mathrm{p}=.03$. Across all patients and trials, we only registered one single key press when a stimulus was presented in a blind field only (P2) and one keypress in a catch trial (P1). In both cases, the RTs could be classified as outliers (RTs > $1250 \mathrm{~ms})$.

Localisation: On group-level a linear regression analysis on the data showed that target eccentricity was a highly significant predictor of pointing distance in the sighted visual fields of the patients, $\mathrm{F}(1,16)=215.4, \mathrm{p}<.001, \mathrm{R}^{2}=.93$. In addition, the linear regression 
analysis applied to the individual patient data further confirmed that, as expected, all patients adjusted their pointing position with target eccentricity in their sighted visual field (Figure 3b, all $\mathrm{p}<.001$ ). Regarding the localisation performance in the patients' blind visual field, target distance did not predict pointing position on a group level, $\mathrm{F}(1,16)=0.06, \mathrm{p}=.82, \mathrm{R}^{2}=.003$. Analysis on the individual patient data confirmed that four of the patients did not scale their pointing distance to target eccentricity at all in their blind visual field (all $\mathrm{p}>.11$, Figure $3 \mathrm{~b}$ ). For one patient $(\mathrm{P} 2)$, the regression revealed a significant linear relationship between pointing distance and target eccentricity, $\mathrm{F}(1,28)=6.7, \mathrm{p}=.015$, with an $\mathrm{R}^{2}$ of .19 . However, this relationship was in the opposite direction than predicted, with shorter pointing distances for targets being presented further away from fixation (see Figure 3b). Finally, a significant regression equation was also found for Patient 4, $\mathrm{F}(1,28)=17.4, \mathrm{p}<.001$, with an $\mathrm{R}^{2}$ of .38 indicating that this patient increased pointing distances for targets presented further away from fixation. This suggests that the patient shows some residual visual processing in her blind hemifield. Note that this is the same patient that showed a significant redundant target effect indicating that this patient may have some spared visual abilities.

\section{Insert figure 3 about bere}

\subsection{Obstacle avoidance}

In both tasks, none of the patients ever reported awareness of an obstacle if it was placed in their blind visual field.

\subsubsection{Group statistics}

Single Obstacle Task: The mean lateral changes in hand position (sensitivity) of the patient group and the control group as a result of a change in obstacle position $(10 \mathrm{~cm}$ vs. $6 \mathrm{~cm}$ from the midline) can be seen in Figure 4. A 2 (group: patients, controls) x 2 (visual field: [matched] sighted, [matched] blind) mixed-design ANOVA on sensitivity to obstacle position revealed a main effect of visual field, $F(1,10)=13.32, p=.004$. As there also was a significant interaction between group and visual field, $\mathrm{F}(1,10)=8.31, \mathrm{p}$ $=.016$, the main effect cannot be meaningfully interpreted. Post-hoc analysis confirmed that patients were more sensitive to an obstacle shift occurring in their sighted field (12.4 $\mathrm{mm} \pm 1.5 \mathrm{~mm})$ than their blind field $(0.11 \mathrm{~mm} \pm 2.3 \mathrm{~mm}), \mathrm{t}(5)=3.85, \mathrm{p}=.012$. There was no difference in sensitivity between the two matched visual fields in the control 
sample $(10.3 \mathrm{~mm} \pm 1.7 \mathrm{~mm}$ vs. $8.9 \mathrm{~mm} \pm 2.3 \mathrm{~mm} ; \mathrm{p}=.50)$. Independent samples t-tests revealed a significant difference between patients' blind fields and the matched fields of the control subjects (i.e. right visual fields for control participants 1 to 4 and left visual fields for control participants 5 and 6$), \mathrm{t}(10)=2.69, \mathrm{p}=.023$, but, as expected, no significant difference between patients' sighted fields and the corresponding visual fields of control subjects $(\mathrm{p}=.33)$. Furthermore, a one-sample t-test confirmed that, in their blind visual field, patients' sensitivity to obstacle shifts was not significantly different from zero $(p=.97)$.

As it has been shown that the presence of an obstacle in the working space can also affect MTs (Chapman \& Goodale, 2008, 2010; Mon-Williams, Tresilian, Coppard, \& Carson, 2001), one could argue that MTs may provide a more sensitive measure to detect obstacle avoidance effects than changes in the movement path. Hence, we also tested if participants slowed their movements depending on whether an obstacle was present and depending on its position in the workspace ([matched] intact vs. [matched] blind, averaged across the inner and outer positions). Even though control participants tended to show slightly shorter MTs when no obstacle was present (749 ms $\pm 49 \mathrm{~ms}$ ) than when an obstacle was presented in either visual field (matched intact visual field: $778 \pm 51 \mathrm{~ms}$; matched blind visual field: $762 \mathrm{~ms} \pm 54 \mathrm{~ms}$ ), these differences did not reach significance (all p>.09). Similarly, all patients showed comparable movement times across all conditions (no obstacle: $842 \mathrm{~ms} \pm 69 \mathrm{~ms}$, obstacle in intact field: $857 \mathrm{~ms} \pm 72 \mathrm{~ms}$, obstacle in blind field: $851 \mathrm{~ms} \pm 74 \mathrm{~ms}$, all $\mathrm{p}>.56$ ). Finally, even though patients showed overall slightly slower MTs than the control participants, this difference was not statistically significant $(\mathrm{p}=.34)$.

\section{Insert figure 4 about here}

Two Obstacle Task: The same analysis was conducted on the two-obstacle task. Again there was a main effect of visual field, $\mathrm{F}(1,10)=5.08, \mathrm{p}=.048$, and, more importantly, a significant interaction effect, $\mathrm{F}(1,10)=10.30, \mathrm{p}=.009$, between group and visual field on the sensitivity to obstacle position. Post-hoc analysis confirmed that patients were as in the single-obstacle task - more sensitive to an obstacle shift in their sighted visual field $(10.8 \mathrm{~mm} \pm 1.6 \mathrm{~mm})$ than in their blind field $(1.3 \mathrm{~mm} \pm 1.2 \mathrm{~mm}), \mathrm{t}(5)=3.88, \mathrm{p}=$ .012 (see Figure 4). Again, control subjects' sensitivity did not differ significantly between 
both matched fields $(\mathrm{p}=.53)$. Independent samples t-tests confirmed a significant difference between patients' blind field and the matched visual fields of control subjects, $\mathrm{t}(10)=3.71, \mathrm{p}=.007$, while there was no difference between patients' sighted fields and the matched visual fields of the control subjects $(p=.55)$. Again, patients' performance in their blind visual field was not significantly different from zero $(p=.31)$.

A 2 (group: patients, controls) x 3 (obstacle position: [matched] sighted in, [matched] blind in, both out) mixed-design ANOVA on MTs showed no significant main effect of obstacle position $(\mathrm{p}=.36)$ or group $(\mathrm{p}=.17)$. There was no interaction effect $(\mathrm{p}=.91)$. On average it took patients $877 \mathrm{~ms} \pm 78 \mathrm{~ms}$ to complete the movement and control participants $747 \mathrm{~ms} \pm 43 \mathrm{~ms}$.

\subsubsection{Single patient data}

As averaging data across patients might mask effects on a single subject level (as observed for the RTE and localisation task in which P4 showed some residual visual processing) we also looked at the performance of each patient individually in order to investigate if any of the patients showed positive evidence for intact obstacle avoidance behaviour. Figure 5 (single obstacle condition) and Figure 6 (two obstacle condition) show the lateral hand positions of each of the patients (and matched controls) for every trial and for both visual fields (as well as the mean value across all trials with the same obstacle configuration). For the single obstacle condition, independent t-tests confirmed that all patients adjusted their hand trajectories in response to obstacle position in their intact visual field (sighted in vs. sighted out, all $\mathrm{p}<.0045$, one-tailed). In contrast, in their blind visual field, none of the patients showed any significant adjustments of hand position in the expected direction (blind in vs. blind out, all $\mathrm{p} \geq .11$, one-tailed). Notably, however, one patient (P5) showed a significant adjustment in his blind field ( $\mathrm{p}=.036$, one-tailed) in the opposite direction to what would be predicted by an obstacle avoidance strategy (i.e. moving the hand further toward the obstacle if it was placed at an inner position, see Figure 5, P5). Examples for complete hand movement paths (average across trials) for a patient (P1) and the corresponding control participant in the single obstacle condition are shown in Figure 7. 
The same tests applied to the two obstacle condition revealed that four out of six patients showed significant adjustments in their intact visual field (all $\mathrm{p} \leq .013$, one-tailed) while two patients ( $\mathrm{P} 4[\mathrm{p}=.12]$ and P6 [p=.076], one-tailed) did not significantly alter their hand position depending on obstacle position in their intact field (note that numerically adjustments occurred in the anticipated direction). Regarding the effect of obstacles' position in the blind visual field, none of the patients showed any significant variations in hand position depending on their location (all $\mathrm{p} \geq .10$, one-tailed). In a nutshell, none of the patients showed any significant adjustments to the position of the obstacles on a trial-by-trial basis when presented in their blind visual field while most of them were sensitive to their position if placed in the intact visual field in both conditions. Hence, we did not find any positive evidence for intact obstacle-avoidance performance in any of the tested patients. Similarly, the control participants also showed, with a few exceptions, significant adjustments on a trial-by-trial basis (see Figures 5 and 6). This is in line with the conclusion based on the group data.

\section{Insert figure 7 about bere}

\section{Discussion}

It has been argued that even though conscious visual input from the primary visual pathways is critical for normal perceptual performance, it may not necessarily be required for normal visuomotor performance (Goodale \& Milner, 2004; McIntosh, McClements, Schindler, et al., 2004; Milner \& Goodale, 2006; Striemer et al., 2009). Yet, we know that the ability to process certain stimulus properties such as size, colour, motion, orientation and position can occur after V1-damage and without visual awareness in both the perceptual and the visuomotor domain (e.g., Azzopardi \& Cowey, 2001; Carey, Trevethan, Weiskrantz, \& Sahraie, 2012; Danckert et al., 2003; Danckert \& Rossetti, 2005; Jackson, 1999; Perenin \& Jeannerod, 1975; Perenin \& Rossetti, 1996; Stoerig, 1993; Stoerig \& Cowey, 1997; Weiskrantz et al., 1974; Whitwell et al., 2011). In general, it has been found that for perceptual tasks this so called blindsight performance is well above chance level but still inferior to normal performance in seeing observers. However, this might not be the case for the visuomotor domain. In particular, it has been argued that a lack of awareness of the presence and position of obstacles does not necessarily compromise the associated avoidance behaviour (McIntosh, McClements, Schindler, et al., 2004; Striemer et al., 2009). Hence, Striemer et al. (2009) suggested that the dorsal stream might not critically depend on input from V1. They base their claim largely on the 
observation of one single hemianopic patient (CB) who was able to avoid obstacles placed in his blind visual field. If it is true that, as suggested, the subcortical pathways primarily mediate obstacle avoidance, then we should be able to observe the ability to avoid obstacles quite reliably in patients with circumscribed damage to the occipital cortex. However, this was not found in our study testing a group of patients with visual field defects after selective lesions to the occipital lobes.

On a group-level, we did not find any evidence for spared (let alone normal) obstacle avoidance behaviour in patients for obstacles placed in their blind visual field independent of whether or not a second obstacle was present in the sighted visual field as well. Furthermore, patients' sensitivity to obstacles placed in their blind field was significantly smaller than the sensitivity to obstacle position measured in the control sample for both conditions, confirming that our patients were not able to reliably avoid obstacles placed in their blind visual fields. Interestingly, when looking at each patient's performance individually, we found that one patient who showed reliable signs of blindsight in both the redundant target effect task and the localisation task (P4) did not show any indication for retained obstacle avoidance behaviour. This is intriguing as it was claimed by Striemer and colleagues (2009) that obstacle avoidance may be a more sensitive measure for determining visuomotor blindsight than the localisation task - as their patient had shown intact obstacle avoidance but was unable to point to targets placed in his blind visual field. Our findings provide evidence that the opposite pattern can also be observed suggesting that intact obstacle avoidance is not a prerequisite for retained localisation performance.

To summarise, none of our patients showed reliable evidence for the ability to avoid obstacles placed in their blind field. Given that we took great care to select patients whose lesions were focused on the occipital cortex with sparing of the subcortical visual areas and the dorsal pathways, structures that have been identified to be crucial for the occurrence of action-blindsight (for review see, Danckert \& Rossetti, 2005; Rabbo, Koch, Lefèvre, \& Seizeur, 2015; Sincich, Park, Wohlgemuth, \& Horton, 2004), it seems likely that subcortical visual input to dorsal stream areas is insufficient to generate normal obstacle avoidance behaviour. Furthermore, the fact that one of our patients (P4) could successfully point to targets but was unable to avoid obstacles in her blind field refutes the hypothesis that obstacle-avoidance is less dependent on visual input from occipital areas than pointing. 
These findings have implications for the perception-action model, in particular the proposed role of subcortical visual pathways for the control of visuomotor functions. It has been known for some time that blindsight performance in patients with V1-lesions may rely on pathways that bypass V1, but while blindsight performance in perceptual tasks seems to be merely above chance and not within the normal range, it has been suggested that blindsight performance in visuomotor tasks can be as good as that observed in healthy people. This preserved visuomotor behaviour despite V1-damage has been presented as strong evidence that also normal obstacle avoidance behaviour does not rely on V1-input. In contrast, the above-chance blindsight performance as typically observed in perceptual tasks, has been suggested to illustrate the brain's remarkable ability to make use of any information available to compensate for the loss of the routes that are normally involved in solving these tasks. In other words, cases of actionblindsight showing similar performance levels as those found in neurologically healthy controls have been interpreted as evidence that dorsal stream functions are generally less reliant on V1-input than ventral stream functions. This qualitative difference between ventral and dorsal processing is in line with the predictions of the perception-action model. However, the situation is complicated by the fact that there are some types of visuomotor behaviour such as grasping (Whitwell et al., 2011) and pointing (e.g., Corbetta et al., 1990; Danckert et al., 2003; Perenin, Ruel, \& Hecaen, 1980; Striemer et al., 2009) which were found to suffer from V1-damage in a similar way as perceptual tasks. This contradiction could be resolved by characterising the dorsal stream as a collection of parallel sensorimotor loops. Some sensorimotor loops underlying for example grasping and pointing may take their visual input from the cortical sources, and in particular V1, while others like the loop underlying obstacle-avoidance behaviour may take their input from subcortical sources. If this was true, we would expect that obstacleavoidance behaviour is always less affected by occipital damage than pointing, grasping or perceptual tasks. However, our results contradict this notion. In our study obstacleavoidance was highly impaired for all patients while pointing and redundant target performance was spared in one of them. Consequently, our study provides no neuropsychological evidence to support the claim that V1, or more generally early visual areas, are less involved in dorsal than in ventral stream functions. Hence, obstacleavoidance does not seem to provide a more sensitive measure for revealing residual visual processing after occipital damage. Instead, preserved obstacle avoidance appears at 
least as hard to find as most other perceptual blindsight phenomena (Blythe, Kennard, \& Ruddock, 1987; Marzi et al., 1986; Schärli, Harman, \& Hogben, 1999).

Importantly, our findings also highlight that there are considerable individual differences in blindsight performance between patients who do not necessarily differ hugely with respect to their lesion locations. For instance, it seems hard to explain why we found some spared ability of using residual vision for pointing in a patient (P4) who suffered from a more extensive lesion to the visual cortex than Striemer's patient CB. Quite possibly such variations may reflect differences in how patients have learnt to use their residual visual capacities. This has important implications as it shows that when we measure behavioural dissociations between neurological patients, we have to be aware of the possibility that differences in behavioural capacity may not necessarily relate to, or be accounted for by differences in their pathology. Hence, patient CB's ability to avoid obstacles in his blind field might also just reflect an idiosyncratic strategy of using his remaining visual capacities that he had acquired prior to being tested in the experiment.

Alternatively, one could argue that we did not observe spared obstacle avoidance behaviour as our patients suffered generally from more extensive lesions than patient CB. As pointed out above, we only included patients in the study whose lesions were focussed on the occipital cortex and spared subcortical structures. Only two of our patients had slightly more extensive lesions (P1 and P4). In both patients, lesions came close to the occiptio-temporo-parietal junction, i.e. putatively area V5/MT. However, based on previous reports on coordinates of area V5/MT (Dumoulin et al., 2000; Sunaert, Van Hecke, Marchal, \& Orban, 1999; Watson et al., 1993) we considered substantial damage to this area as unlikely.

Furthermore, Patient 1 showed a Riddoch phenomenon which is indicative of preserved functioning of area V5/MT. Yet, given that lesions between patients are never identical, it is possible that CB's ability for obstacle-avoidance was mediated by cortical structures that have been damaged in our patients. At this point we can, however, only speculate about which cortical areas may be relevant for the spared obstacle-avoidance behaviour. Based on the limited lesion information provided for patient CB (Figure 1 in Striemer et al., 2009) it appears that his lesion was largely restricted to V1. This is further supported by the fact that patient CB showed a Charles-Bonnet-Syndrom (i.e. visual hallucinations in this blind visual field) which was found to occur more often in hemianopic patients with smaller lesions of the occipital cortices that spare the visual association areas (Lance, 
1976; Menon, Rahman, Menon, \& Dutton, 2003; Vaphiades, Celesia, \& Brigell, 1996). Even though extension to adjacent visual areas seems very likely, the lesions of most of our patients' lesions were more extensive and mostly expanded into the visual association cortex (areas V2 and V3, compare Figure 1). While it was previously assumed that the prestriate area V2 as well as areas V3 and V4 are silenced after V1 lesions (Girard \& Bullier, 1989; Girard, Salin, \& Bullier, 1991), more recent studies on non-human primates suggest that these areas can remain responsive after damage to V1 (Schmid, Panagiotaropoulos, Augath, Logothetis, \& Smirnakis, 2009; Schmid et al., 2013). Furthermore, neurons from the LGN were found to send visual stimulus information directly to V2 and other functional areas of the occipital lobe bypassing V1 (Schmid et al., 2010). Based on these findings, it has been hypothesised that the retained, albeit greatly reduced, neural activity in areas V2 and V3 after V1 damage may indeed contribute to the blindsight phenomenon (Schmid et al., 2009; for review see also, Silvanto, 2015). However, it appears not plausible to assume that highly attenuated residual activity in visual associative areas drives action-blindsight substantially.

In addition to interindividual differences in lesion anatomy, it needs to be pointed out that there were also some differences between the exact obstacle designs implemented in our study and in the study conducted by Striemer and colleagues. Specifically, Striemer et al. (2009) used comparably large obstacles in their task (base of $4 \times 4 \mathrm{~cm}$ with a height of $25 \mathrm{~cm}$ ) that were placed further away from the midline (10 and $15 \mathrm{~cm}$ respectively). In contrast, our obstacles were much smaller (base of $1 \times 1 \mathrm{~cm}$ and a height of $15 \mathrm{~cm}$ ) but also placed a bit nearer to the midline $(6$ and $10 \mathrm{~cm})$. However, we think that it is unlikely that these differences can explain why we did not observe preserved obstacle avoidance in our patients' blind fields for two reasons. Firstly, our obstacles still evoked reliable avoidance effects in the patients' intact visual fields as well as in the control participants. Secondly, and related to the first point, the sensitivity patient CB showed for a $50 \mathrm{~mm}$ obstacle shift in his sighted field (i.e. a $15.8 \mathrm{~mm}$ shift in hand position that corresponds to $31.6 \%$ of the distance the obstacle was moved by) is quite similar to the sensitivity we observed for a $40 \mathrm{~mm}$ single obstacle shift in the sighted fields of our patients (a 12.4 $\mathrm{mm}$ shift in hand position that corresponds to $31 \%$ of the distance the obstacle was moved by).

Finally, it cannot be excluded that the observations reported by Striemer et al. (2009) may simply reflect a chance finding. Normal obstacle avoidance behaviour was observed in only one session for only one patient. For such a small sample of observations a few 
outliers can be sufficient to generate an effect. In fact, our own study provides an interesting example for such a chance finding, with Patient 5 responding consistently to the position of an obstacle placed in his blind field in the single-obstacle condition. Except that in this case, the patient responded in exactly the opposite direction to what one would predict, i.e. his reaching trajectory shifted towards the obstacle (see Figure 5, first row). This behaviour is quite counter-intuitive and therefore it is easy to dismiss the finding as spurious. It also illustrates nicely that significant findings are not always reliable, especially if conclusions are made based on a small number of observations. Thus, we think that in order to establish conclusively whether or not a patient shows a distinct blindsight phenomenon, it is necessary to test their performance in several sessions to replicate the observation (see Corbetta et al., 1990 for a similar argument). This seems particularly important for visuomotor tasks as performance in these tasks tends to be very variable even within neurologically intact participants (see $2^{\text {nd }}$ row in Figures 5 and 6).

Before we conclude, it is important to stress the fact that we do not suggest that obstacle avoidance is generally impossible without V1. There is a classical monkey study (Humphrey, 1974) as well as a recent neuropsychological study on a patient with bilateral damage to V1 (De Gelder et al., 2008) both providing evidence that obstacles can be avoided after extensive damage to the primary visual pathways and without conscious visual experience during locomotion. Hence similar mechanisms may also underlie sensitivity of hand movements to obstacles during reaching. However, there are also some crucial differences between locomotion paradigms and the hand movement tasks as employed in the current study. Most obviously, locomotion causes optic flow, i.e. motion patterns that are processed in the MT/MST complex (Morrone et al., 2000; Smith, Wall, Williams, \& Singh, 2006). This is relevant as recent studies have highlighted the importance of the LGN-MT pathway for the occurrence of perceptual blindsight (Ajina, Pestilli, Rokem, Kennard, \& Bridge, 2015; Tamietto \& Morrone, 2016). Hence, one could hypothesise that action-blindsight too, is more likely to be observed in situations in which motion signals are present. This was clearly not the case in our study as obstacles were stationary and participants did not look at their hand during the movement due to the required fixation. On the other hand, this argument can also not account for the findings of Striemer and colleagues (2009) as they actually occluded vision at movement onset preventing any kind of residual visual feedback to be processed. 
In conclusion, our finding that in a group of six patients with relatively localised lesions in the occipital lobe, none of them showed sensitivity to unseen obstacles in their blind visual field indicates that, at least in most cases, the dorsal pathways seem to critically depend on visual input mediated by the primary visual pathways (see also Hesse et al. 2012). We know from monkey as well as human studies that the dorsal areas that deal with obstacle avoidance do obtain input from subcortical structures via the RTP-pathway (Danckert \& Rossetti, 2005; Kaas \& Lyon, 2007; Lyon et al., 2010; Rodman et al., 1990; Stoerig \& Cowey, 2007; Tamietto et al., 2009; Zihl, 1980). However whether or not this input becomes functionally relevant seems to vary from case to case and may depend on the exact lesion anatomy of the patient (for discussion see, Danckert \& Rossetti, 2005). Considering this, we think that it is unlikely that, as hypothesised by Striemer et al. (2009), complex everyday behaviour such as obstacle avoidance is primarily mediated by the primitive subcortical pathways. Instead we conclude that there is currently no compelling neuropsychological evidence supporting the suggestion that V1-input is less relevant for dorsal than for ventral stream functions. 


\section{References}

Ajina, S., Pestilli, F., Rokem, A., Kennard, C., \& Bridge, H. (2015). Human blindsight is mediated by an intact geniculo-extrastriate pathway. eLife, 4, e08935.

Azzopardi, P., \& Cowey, A. (1997). Is blindsight like normal, near-threshold vision? Proceedings of the National Academy of Sciences, 94(25), 14190-14194.

Azzopardi, P., \& Cowey, A. (2001). Motion discrimination in cortically blind patients. Brain, 124(1), 30-46.

Blythe, I. M., Kennard, C., \& Ruddock, K. H. (1987). Residual Vision in Patients with Retrogeniculate Lesions of the Visual Pathways. Brain, 110, 887-905.

Brainard, D. H. (1997). The psychophysics toolbox. Spatial Vision, 10(4), 433-436.

Bridge, H., Leopold, D. A., \& Bourne, J. A. (2016). Adaptive Pulvinar Circuitry Supports Visual Cognition. Trends in cognitive sciences, 20(2), 146-157.

Carey, D. P., Trevethan, C. T., Weiskrantz, L., \& Sahraie, A. (2012). Does delay impair localisation in blindsight? Neuropsychologia, 50(14), 3673-3680.

Chapman, C. S., \& Goodale, M. A. (2008). Missing in action: the effect of obstacle position ans size on avoidance while reaching. Experimental Brain Research, 191, 83-97.

Chapman, C. S., \& Goodale, M. A. (2010). Seeing all the obstacles in your way: the effect of visual feedback and visual feedback schedule on obstacle avoidance while reaching. [Article]. Experimental Brain Research, 202(2), 363-375. doi: 10.1007/s00221-009-2140-7

Corbetta, M., Marzi, C., Tassinari, G., \& Aglioti, S. (1990). Effectiveness of different task paradigms in revealing blindsight. Brain, 113(3), 603-616.

Cornelissen, F. W., Peters, E. M., \& Palmer, J. (2002). The Eyelink Toolbox: Eye tracking with MATLAB and the psychophysics toolbox. Behavior Research Methods Instruments \& Computers, 34(4), 613-617.

Danckert, J., Revol, P., Pisella, L., Krolak-Salmon, P., Vighetto, A., Goodale, M. A., \& Rossetti, Y. (2003). Measuring unconscious actions in action-blindsight: exploring the kinematics of pointing movements to targets in the blind field of two patients with cortical hemianopia. Neuropsychologia, 41(8), 1068-1081.

Danckert, J., \& Rossetti, Y. (2005). Blindsight in action: what can the different sub-types of blindsight tell us about the control of visually guided actions? [Review].

Neuroscience and Biobehavioral Reviews, 29(7), 1035-1046. doi: 10.1016/j.neubiorev.2005.02.001

De Gelder, B., Tamietto, M., Van Boxtel, G., Goebel, R., Sahraie, A., Van den Stock, J., . . Pegna, A. (2008). Intact navigation skills after bilateral loss of striate cortex. Current Biology, 18(24), R1128-R1129.

Dumoulin, S. O., Bittar, R. G., Kabani, N. J., Baker, C. L., Le Goualher, G., Pike, G. B., \& Evans, A. C. (2000). A new anatomical landmark for reliable identification of human area V5/MT: a quantitative analysis of sulcal patterning. Cerebral Cortex, 10(5), 454-463.

Esterman, B. (1982). Functional scoring of the binocular field. Ophthalmology, 89(11), 1226-1234.

Franz, V. H. (2004). The Optotrak Toolbox. Retrieved April, 15, 2010.

Girard, P., \& Bullier, J. (1989). Visual activity in area V2 during reversible inactivation of area 17 in the macaque monkey. Journal of neurophysiology, 62(6), 1287-1302.

Girard, P., Salin, P. A., \& Bullier, J. (1991). Visual activity in areas V3a and V3 during reversible inactivation of area $\mathrm{V} 1$ in the macaque monkey. Journal of Neurophysiology, 66(5), 1493-1503. 
Goodale, M. A., \& Milner, A. D. (2004). Sight Unseen: An Exploration of Conscious and Unconscious Vision: Oxford: Oxford University Press.

Halligan, P. W., Cockburn, J., \& Wilson, B. (1991). The behavioral assessment of visual neglect. Neuropsychological Rebabilitation, 1(1), 5-32.

Hesse, C., Lane, A. R., Aimola, L., \& Schenk, T. (2012). Pathways involved in conscious vision contribute to obstacle-avoidance behaviour. European Journal of Neuroscience, 36(3), 2383-2390.

Humphrey, N. K. (1974). Vision in a monkey without striate cortex: a case study. Perception, 3(3), 241-255.

Jackson, S. R. (1999). Pathological perceptual completion in hemianopia extends to the control of reach - to - grasp movements. Neuroreport, 10(12), 2461-2466.

Kaas, J. H., \& Lyon, D. C. (2007). Pulvinar contributions to the dorsal and ventral streams of visual processing in primates. Brain Research Reviews, 55(2), 285-296.

Lance, J. W. (1976). Simple formed hallucinations confined to the area of a specific visual field defect. Brain: a journal of neurology, 99(4), 719-734.

Lyon, D. C., Nassi, J. J., \& Callaway, E. M. (2010). A Disynaptic Relay from Superior Colliculus to Dorsal Stream Visual Cortex in Macaque Monkey. Neuron, 65(2), 270-279. doi: http://dx.doi.org/10.1016/i.neuron.2010.01.003

Marzi, C., Tassinari, G., Aglioti, S., \& Lutzemberger, L. (1986). Spatial summation across the vertical meridian in hemianopics: A test of blindsight. Neuropsychologia, 24(6), 749-758.

McIntosh, R. D., McClements, K. I., Dijkerman, H. C., Birchall, D., \& Milner, A. D. (2004). Preserved obstacle avoidance during reaching in patients with left visual neglect. [Article]. Neuropsychologia, 42(8), 1107-1117. doi: 10.1016/j.neuropsychologia.2003.11.023

McIntosh, R. D., McClements, K. I., Schindler, I., Cassidy, T. P., Birchall, D., \& Milner, A. D. (2004). Avoidance of obstacles in the absence of visual awareness. [Article]. Proceedings of the Royal Society of London Series B-Biological Sciences, 271(1534), 15-20. doi: 10.1098/rspb.2003.2545

Menon, G. J., Rahman, I., Menon, S. J., \& Dutton, G. N. (2003). Complex visual hallucinations in the visually impaired: the Charles Bonnet Syndrome. Survey of ophthalmology, 48(1), 58-72.

Milner, A. D., \& Goodale, M. A. (1995). The visual brain in action: Oxford: Oxford University Press.

Milner, A. D., \& Goodale, M. A. (2006). The visual brain in action. (2nd ed.). Oxford: Oxford University Press.

Milner, A. D., \& Goodale, M. A. (2008). Two visual systems re-viewed. [Article]. Neuropsychologia, 46(3), 774-785. doi: 10.1016/j.neuropsychologia.2007.10.005

Mon-Williams, M., Tresilian, J. R., Coppard, V. L., \& Carson, R. G. (2001). The effect of obstacle position on reach-to-grasp movements. Experimental Brain Research, 137, 497-501.

Morland, A. B., Jones, S. R., Finlay, A. L., Deyzac, E., Lê, S., \& Kemp, S. (1999). Visual perception of motion, luminance and colour in a human hemianope. Brain, 122(6), 1183-1198. doi: 10.1093/brain/122.6.1183

Morrone, M., Tosetti, M., Montanaro, D., Fiorentini, A., Cioni, G., \& Burr, D. (2000). A cortical area that responds specifically to optic flow, revealed by fMRI. Nature neuroscience, 3(12), 1322-1328.

Perenin, M., \& Jeannerod, M. (1975). Residual vision in cortically blind hemiphields. Neuropsychologia, 13(1), 1-7.

Perenin, M., \& Rossetti, Y. (1996). Grasping without form discrimination in a hemianopic field. Neuroreport, 7(3), 793-797. 
Perenin, M. T., Ruel, J., \& Hecaen, H. (1980). Residual visual capacities in a case of cortical blindness. Cortex, 16(4), 605-612.

Ptito, A., Lassonde, M., Leporé, F., \& Ptito, M. (1987). Visual discrimination in hemispherectomized patients. Neuropsychologia, 25(6), 869-879.

Ptito, A., Lepore, F., Ptito, M., \& Lassonde, M. (1991). Target detection and movement discrimination in the blind field of hemispherectomized patients. Brain, 114(1), 497-512.

Rabbo, F. A., Koch, G., Lefèvre, C., \& Seizeur, R. (2015). Direct geniculo-extrastriate pathways: a review of the literature. Surgical and Radiologic Anatomy, 37(8), 891-899.

Rice, N. J., Edwards, M. G., Schindler, I., Punt, T. D., McIntosh, R. D., Humphreys, G. W., ... Milner, A. D. (2008). Delay abolishes the obstacle avoidance deficit in unilateral optic ataxia. Neuropsychologia, 46(5), 1549-1557.

Rice, N. J., McIntosh, R. D., Schindler, I., Mon-Williams, M., Démonet, J. F., \& Milner, D. A. (2006). Intact automatic avoidance of obstacles in patients with visual form agnosia. Experimental Brain Research, 174, 176-188.

Rodman, H. R., Gross, C. G., \& Albrigh, T. D. (1990). Afferent basis of visual response properties in area MT of the macaque. II. Effects of superior colliculus removal. Journal of Neuroscience, 10(4), 1154-1164.

Rorden, C., \& Brett, M. (2000). Stereotaxic display of brain lesions. Behavioural neurology, 12(4), 191-200.

Ross, A. I., Schenk, T., \& Hesse, C. (2014). Line-bisectioning and obstacle avoidance: Evidence for separate strategies. Acta psychologica, 151, 74-82.

Schärli, H., Harman, A. M., \& Hogben, J. H. (1999). Blindsight in subjects with homonymous visual field defects. Journal of Cognitive Neuroscience, 11(1), 52-66.

Schindler, I., Rice, N. J., McIntosh, R. D., Rossetti, Y., Vighetto, A., \& Milner, A. D. (2004). Automatic avoidance of obstacles is a dorsal stream function: Evidence from optic ataxia. Nature Neuroscience, 7(7), 779-784.

Schmid, M. C., Mrowka, S. W., Turchi, J., Saunders, R. C., Wilke, M., Peters, A. J., . . Leopold, D. A. (2010). Blindsight depends on the lateral geniculate nucleus. Nature, 466(7304), 373-377.

Schmid, M. C., Panagiotaropoulos, T., Augath, M. A., Logothetis, N. K., \& Smirnakis, S. M. (2009). Visually driven activation in macaque areas V2 and V3 without input from the primary visual cortex. Plos One, 4(5), e5527.

Schmid, M. C., Schmiedt, J. T., Peters, A. J., Saunders, R. C., Maier, A., \& Leopold, D. A. (2013). Motion-sensitive responses in visual area V4 in the absence of primary visual cortex. The Journal of Neuroscience, 33(48), 18740-18745.

Silvanto, J. (2015). Why is "blindsight" blind? A new perspective on primary visual cortex, recurrent activity and visual awareness. Consciousness and cognition, 32, 1532.

Sincich, L. C., Park, K. F., Wohlgemuth, M. J., \& Horton, J. C. (2004). Bypassing V1: a direct geniculate input to area MT. Nature neuroscience, 7(10), 1123-1128.

Smith, A., Wall, M., Williams, A., \& Singh, K. D. (2006). Sensitivity to optic flow in human cortical areas MT and MST. European Journal of Neuroscience, 23(2), 561-569.

Stoerig, P. (1993). Sources of blindsight. Science, 261(5120), 493-494.

Stoerig, P., \& Cowey, A. (1997). Blindsight in man and monkey. [Review]. Brain, 120, 535-559.

Stoerig, P., \& Cowey, A. (2007). Blindsight. Current Biology, 17(19), R822-R824. doi: http://dx.doi.org/10.1016/j.cub.2007.07.016

Striemer, C. L., Chapman, C. S., \& Goodale, M. A. (2009). Real-time obstacle avoidance in the absence of primary visual cortex. Proceedings of the National Academy of Sciences of the United States of America, 106(37), 15996-16001. 
Sunaert, S., Van Hecke, P., Marchal, G., \& Orban, G. A. (1999). Motion-responsive regions of the human brain. Experimental brain research, 127(4), 355-370.

Talairach, J., \& Tournoux, P. (1988). Co-planar stereotaxic atlas of the human brain. 3Dimensional proportional system: an approach to cerebral imaging. New York: Thieme Medical.

Tamietto, M., Cauda, F., Corazzini, L. L., Savazzi, S., Marzi, C. A., Goebel, R., . . . de Gelder, B. (2009). Collicular Vision Guides Nonconscious Behavior. Journal of Cognitive Neuroscience, 22(5), 888-902. doi: 10.1162/jocn.2009.21225

Tamietto, M., \& Morrone, M. C. (2016). Visual Plasticity: Blindsight Bridges Anatomy and Function in the Visual System. Current Biology, 26(2), R70-R73.

Vaphiades, M. S., Celesia, G. G., \& Brigell, M. G. (1996). Positive spontaneous visual phenomena limited to the hemianopic field in lesions of central visual pathways. Neurology, 47(2), 408-417.

Walsh, T. J. (2011). Visual fields: Examination and Interpretation (3rd ed.). Oxford: University Press.

Ward, R., \& Jackson, S. R. (2002). Visual attention in blindsight: sensitivity in the blind field increased by targets in the sighted field. NeuroReport, 13(3), 301-304.

Watson, J. D. G., Myers, R., Frackowiak, R. S. J., Hajnal, J. V., Woods, R. P., Mazziotta, J. C., . . Zeki, S. (1993). Area V5 of the human brain: evidence from a combined study using positron emission tomography and magnetic resonance imaging. Cerebral Cortex, 3(2), 79-94.

Weiskrantz, L., Warrington, E. K., Sanders, M., \& Marshall, J. (1974). Visual capacity in the hemianopic field following a restricted occipital ablation. Brain, 97(1), 709728.

Whitwell, R. L., Striemer, C. L., Nicolle, D. A., \& Goodale, M. A. (2011). Grasping the non-conscious: Preserved grip scaling to unseen objects for immediate but not delayed grasping following a unilateral lesion to primary visual cortex. Vision research, 51(8), 908-924.

Zihl, J. (1980). "Blindsight": Improvement of visually guided eye movements by systematic practice in patients with cerebral blindness. Neuropsychologia, 18(1), 71 78. doi: http://dx.doi.org/10.1016/0028-3932(80)90085-8 


\section{Figure Legends}

Fig. 1. Lesion plots for all six patients. Based on available clinical imaging lesions were mapped on a standard template (Montreal Neurological Institute brain). Talairach ₹coordinates (Talairach \& Tournoux, 1988) of each transverse section are given.

Fig. 2. Schematic diagram of the obstacle setup (not drawn to exact scale).

Fig. 3. (a) Redundant target effect: RTs for targets presented in the sighted field only or the sighted and blind field simultaneously for each patient. (b) Localisation task: Horizontal distance of pointing location from fixation in both the intact and the blind visual field of each patient. Error bars indicate standard deviations within patients.

Fig. 4. The mean lateral changes in hand position (sensitivity) of the patient group and age-matched control group $(\mathrm{N}=6)$, as a result of a $40 \mathrm{~mm}$ shift of the left or right obstacles, for both the single obstacle and two obstacles tasks. Error bars depict standard errors of mean (SEM) between subjects.

Fig. 5. The lateral hand position as measured on a trial-by-trial basis (and the corresponding mean values) for each of the obstacle positions in the single obstacle task for all patients and the corresponding control participants. Asterisks in brackets indicate significant difference between trials. Note that P1 to P4 were suffering from right visual field defects, hence the performance in their blind field should be compared to that of matched control subjects in their right visual fields. In contrast, P5 and P6 were suffering from left visual field defects, so the avoidance performance in their blind visual field should be compared to the performance of the matched controls in the left visual field. For healthy participants, obstacles placed in the right visual field tend to yield slightly larger avoidance effects as they are more obstructive to the movement.

Fig. 6. The lateral hand position as measured on a trial-by-trial basis (and the corresponding mean values) for each of the obstacle positions in the two-obstacle task for all patients and the corresponding control participants. Asterisks in brackets indicate significant difference between trials. Note that P1 to P4 were suffering from right visual field defects, hence the performance in their blind field should be compared to that of matched control subjects in their right visual fields. In contrast, P5 and P6 were suffering 
from left visual field defects, so the avoidance performance in their blind visual field should be compared to the performance of the matched controls in the left visual field. For healthy participants, obstacles placed in the right visual field tend to yield slightly larger avoidance effects as they are more obstructive to the movement.

Fig 7. A representative, time-normalised hand trajectory plot (averaged across trials) as recorded in the single obstacle task (upper row) and the two obstacles task (lower row) for Patient 1 who suffered from right-quadranopia (left panels) and the corresponding control participant (right panels). Obstacles were placed at $200 \mathrm{~mm}$ in y-direction. 

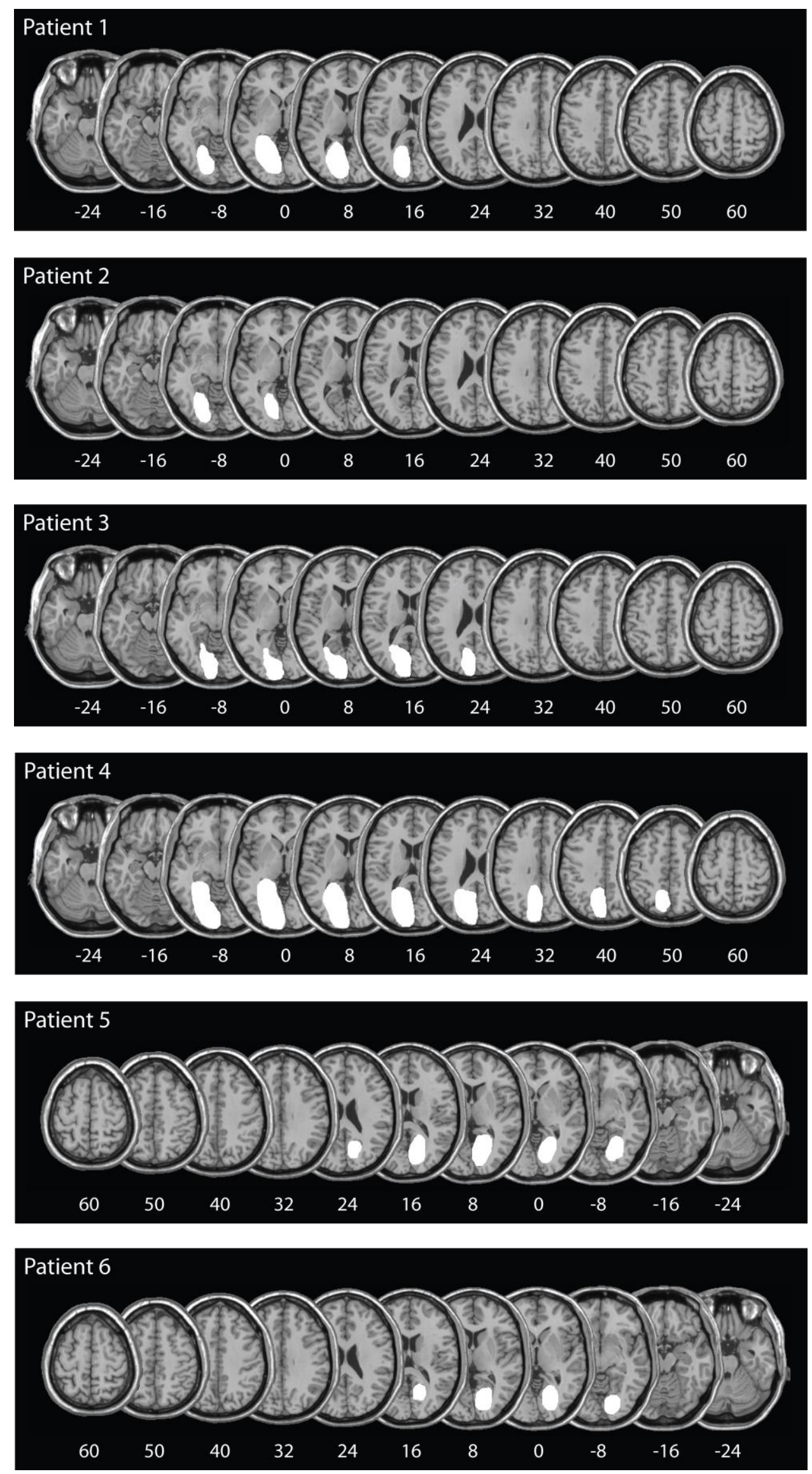

Figure 1 


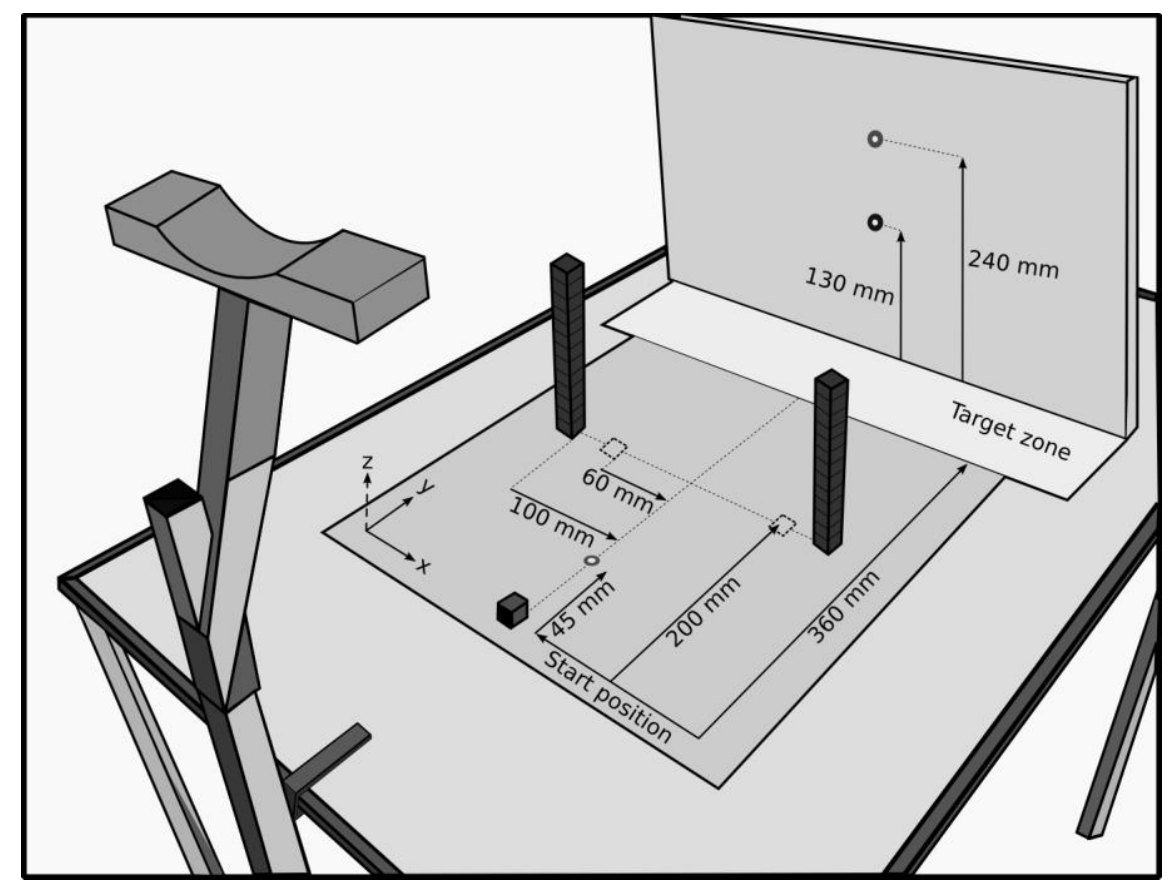

Figure 2 
a)

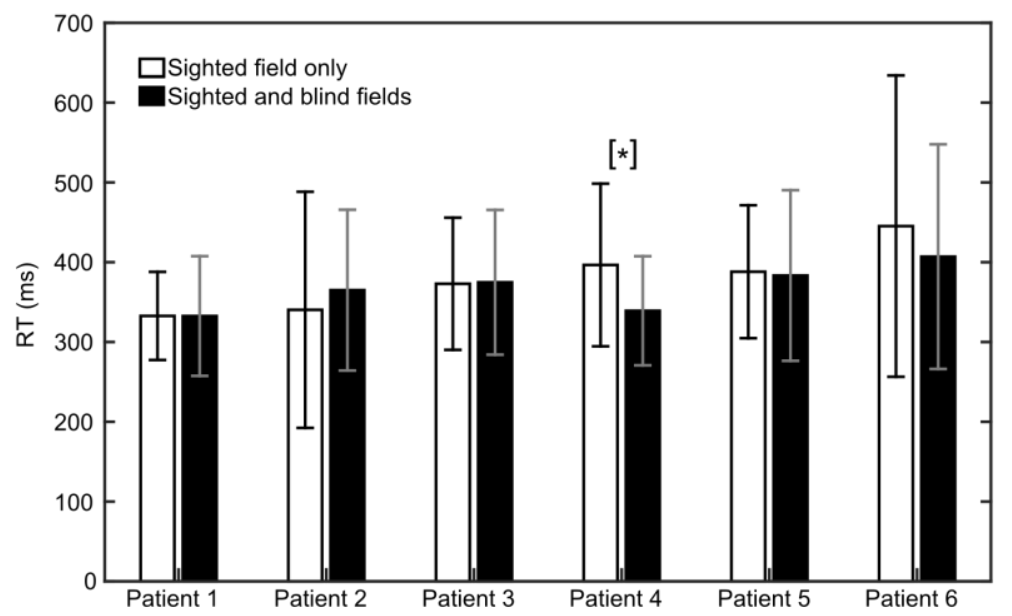

b)
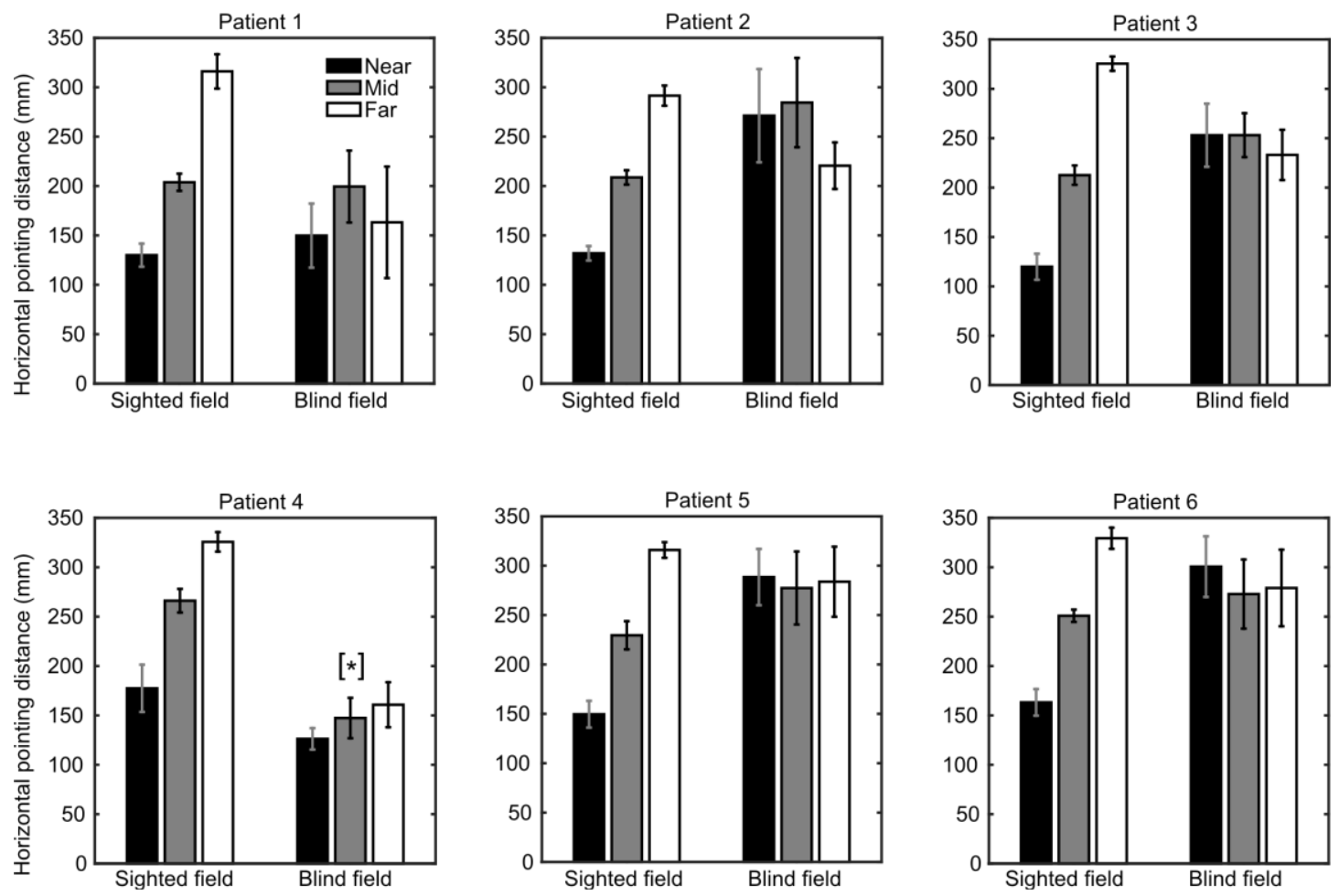

Figure 3 


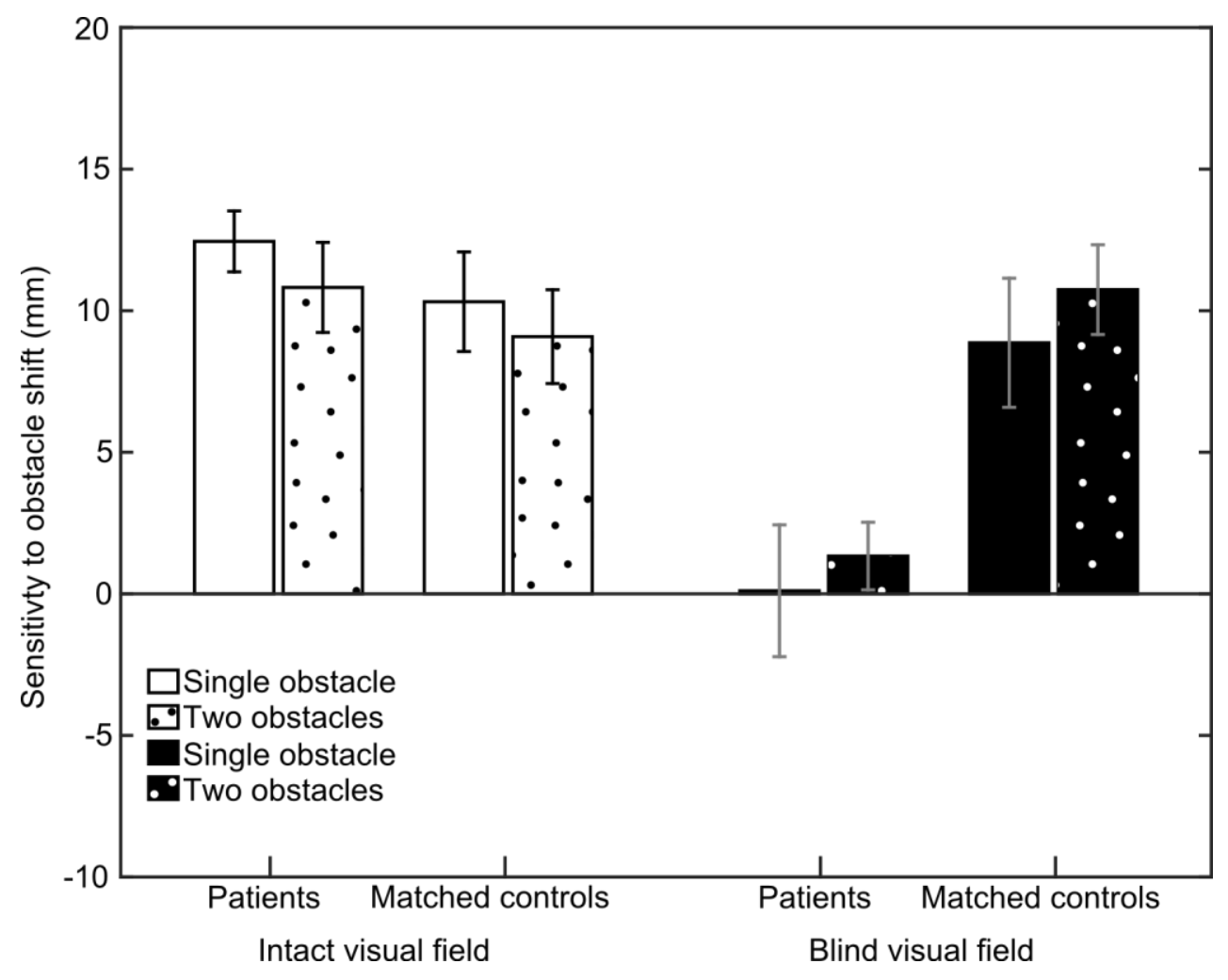

Figure 4 

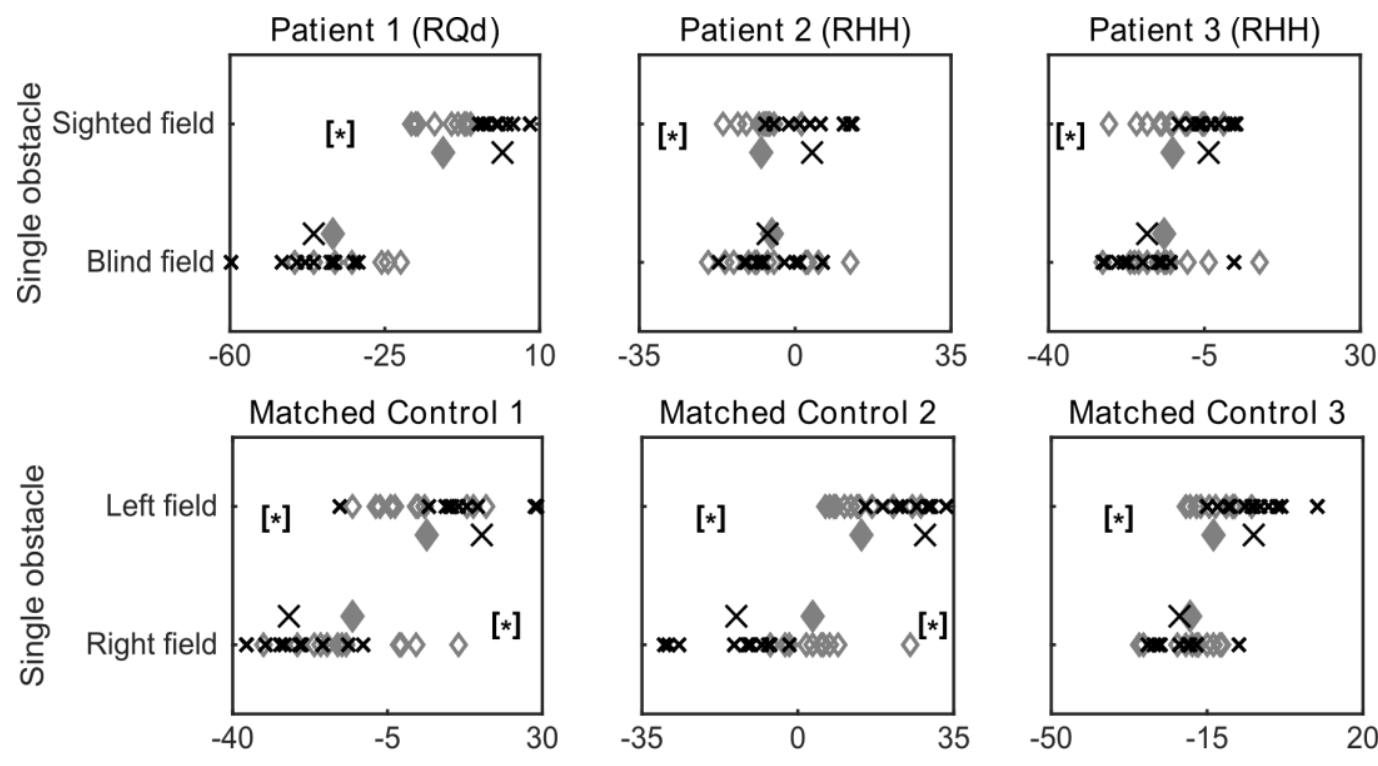

Mean: obstacle at outer position

X Mean: obstacle at inner position

$\diamond$ Single trial: obstacle at outer position

$X$ Single trial: obstacle at inner position

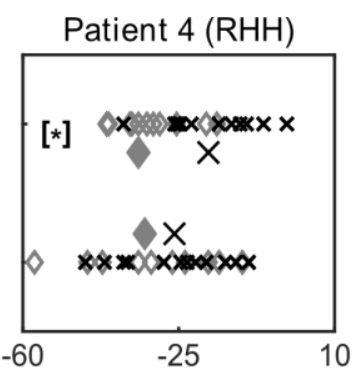

Matched Control 4

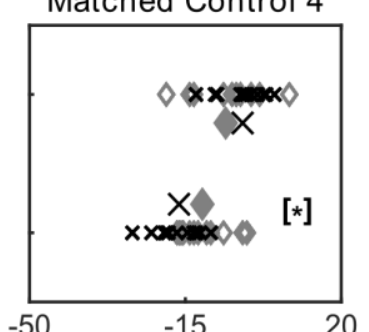

$-15$

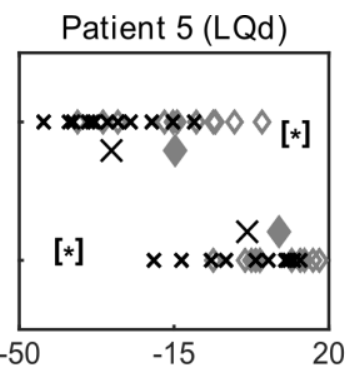

Matched Control 5

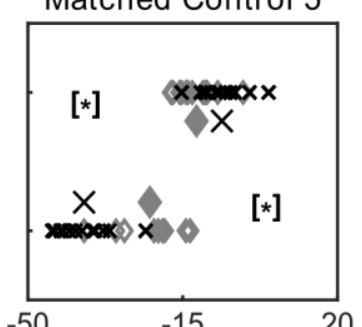

[*] Denotes a significant difference

between obstacle positions within a field

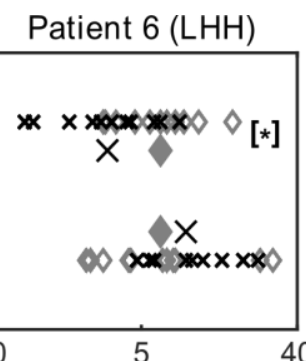

Matched Control 6

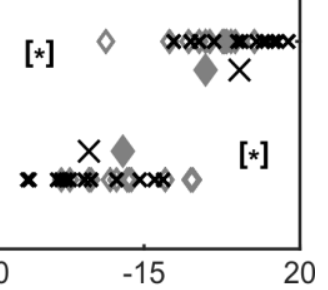

Figure 5 

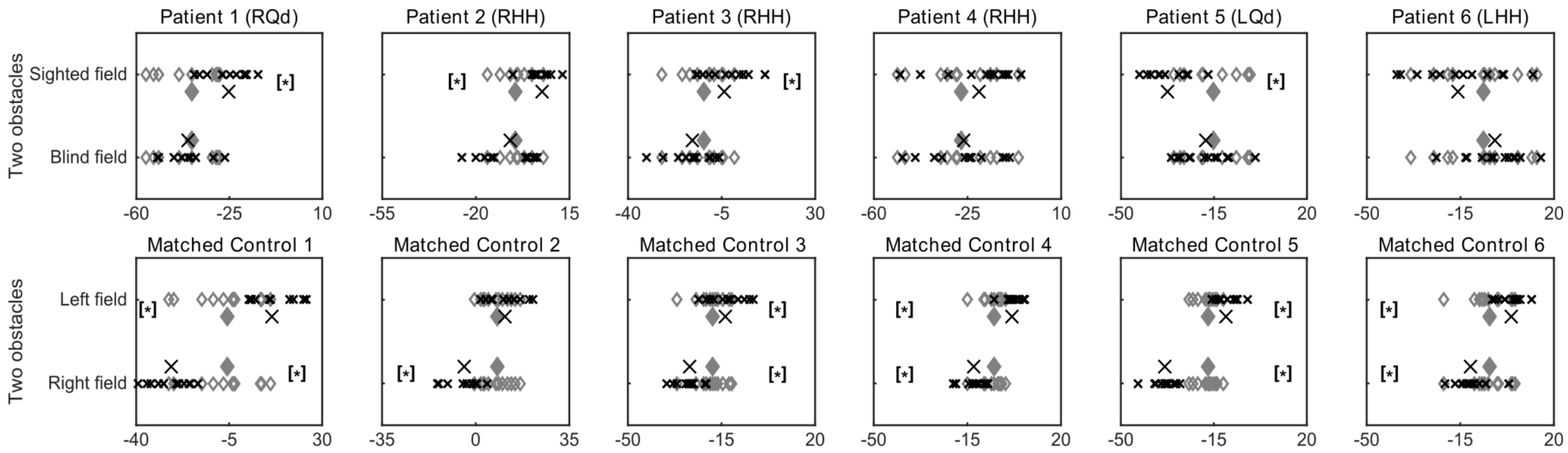

Matched Control 6

lateral hand position in $\mathrm{x}$-direction $(\mathrm{mm})$

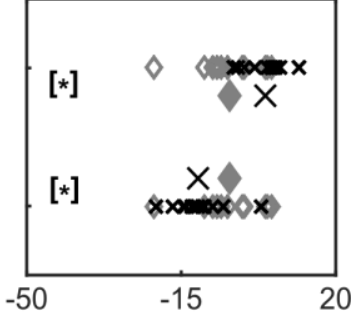

Mean: obstacle at outer position

X Mean: obstacle at inner position

$\diamond$ Single trial: obstacle at outer position

$\times$ Single trial: obstacle at inner position

[*] Denotes a significant difference

between obstacle positions within a field

Figure 6 


\section{Single obstacle}

a)

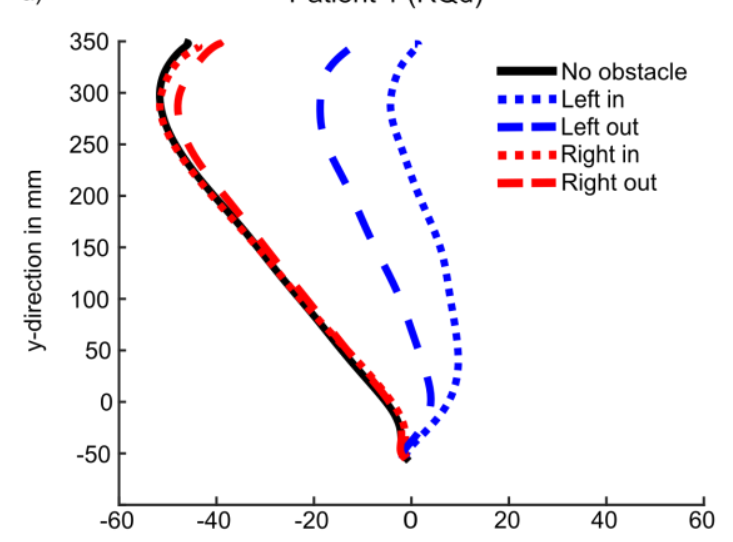

b)

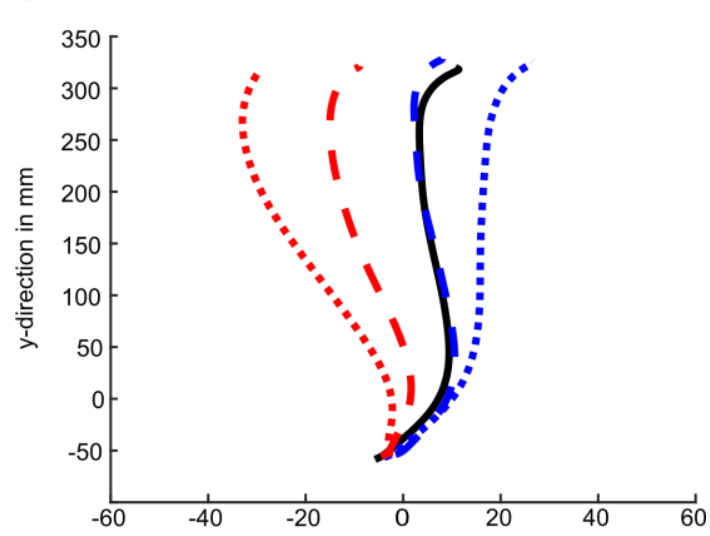

Two obstacles

c)

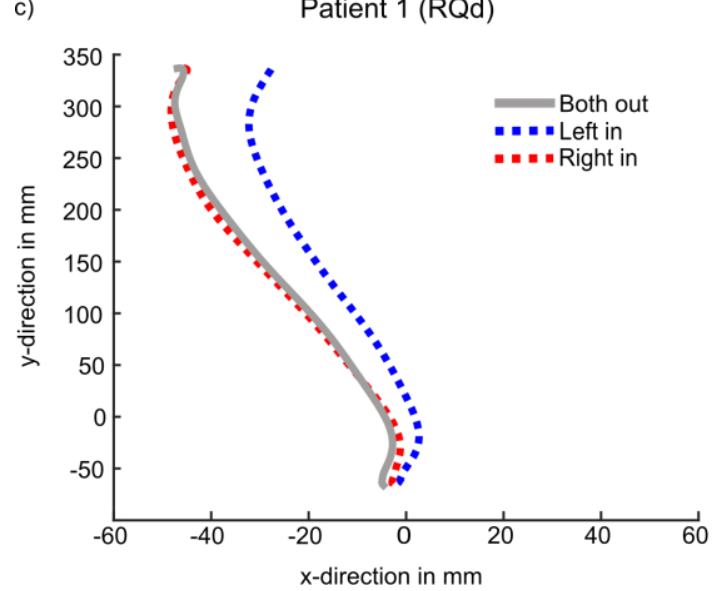

d)

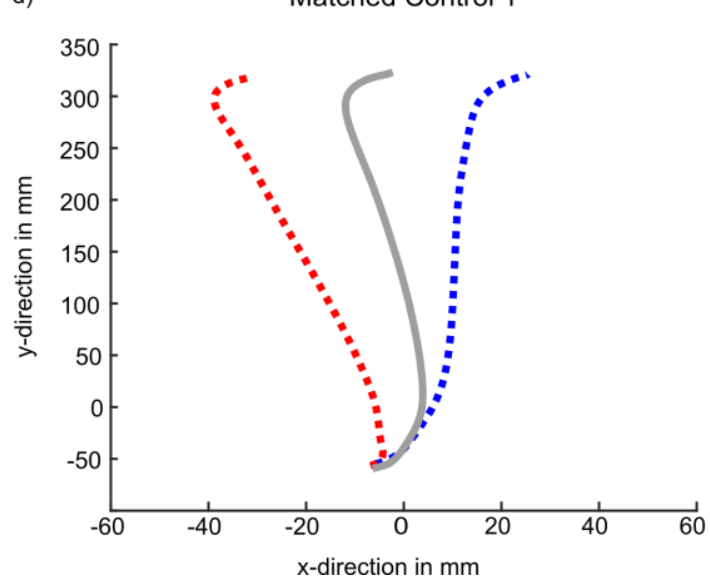

Figure 7 
Table 1. General characteristics of the six patients and their areas of blindness.

\begin{tabular}{|c|c|c|c|c|c|c|}
\hline Patient & $\begin{array}{l}\text { Sex } \\
(\mathrm{M} / \mathrm{F})\end{array}$ & $\begin{array}{l}\text { Age } \\
\text { (years) }\end{array}$ & Side of defect & $\begin{array}{l}\text { Macular } \\
\text { Sparing }\left(^{\circ}\right)\end{array}$ & Lesion location & $\begin{array}{l}\text { Duration } \\
\text { (months) }\end{array}$ \\
\hline P1 & $\mathrm{F}$ & 42 & $\begin{array}{l}\text { Right-superior } \\
\text { quadranopia }\end{array}$ & 2 & $\begin{array}{l}\text { Extensive damage to the left medial } \\
\text { occipital cortex, extending to the } \\
\text { medial occipito-temporal gyrus; } \\
\text { marginal damage to the parieto- } \\
\text { occipital sulcus }\end{array}$ & 14 \\
\hline P2 & M & 60 & $\begin{array}{l}\text { Right } \\
\text { hemianopia }\end{array}$ & $\begin{array}{l}10 \text { (inferior) } \\
3 \text { (superior) }\end{array}$ & $\begin{array}{l}\text { Small lesion to the left medial occipital } \\
\text { cortex, extending to the medial } \\
\text { occipito-temporal gyrus }\end{array}$ & 6 \\
\hline P3 & M & 64 & $\begin{array}{l}\text { Right } \\
\text { hemianopia }\end{array}$ & 0 & $\begin{array}{l}\text { Primary damage to the left medial } \\
\text { occipital cortex,extending to the } \\
\text { medial occipito-temporal gyrus }\end{array}$ & 7 \\
\hline P4 & $\mathrm{F}$ & 73 & $\begin{array}{l}\text { Right } \\
\text { hemianopia }\end{array}$ & 0 & $\begin{array}{l}\text { Extensive damage to the left medial } \\
\text { occipital cortex that extends to the } \\
\text { medial occipito-temporal gyrus as well } \\
\text { as to the parieto-occipital sulcus }\end{array}$ & 7 \\
\hline P5 & M & 63 & $\begin{array}{l}\text { Left-inferior } \\
\text { quadranopia }\end{array}$ & 10 & $\begin{array}{l}\text { Primary damage to the right medial } \\
\text { occipital cortex,extending to the } \\
\text { medial occipito-temporal gyrus }\end{array}$ & 7 \\
\hline P6 & M & 71 & $\begin{array}{l}\text { Left } \\
\text { hemianopia }\end{array}$ & $\begin{array}{l}0 \text { (superior) } \\
8 \text { (inferior) }\end{array}$ & $\begin{array}{l}\text { Primary damage to the right medial } \\
\text { occipital cortex,extending to the } \\
\text { medial occipito-temporal gyrus }\end{array}$ & 10 \\
\hline
\end{tabular}




\section{Acknowledgement}

This work was funded by the RS MacDonald Charitable Trust (awarded to C. Hesse). T. Schenk was supported by a grant from the German Research Council (DFG - SCHE 735/3-1). The authors would like to thank Dr Stefanie Biehl for her valuable advice on lesion localisation based on the CT and MRI scans of the patients. We would also like to thank all the patients for taking part in our experiments and for giving up so much of their free time. 